\title{
Early treatment with ambrisentan of mildly elevated mean pulmonary arterial pressure associated with systemic sclerosis: a randomized, controlled, double-blind, parallel group study (EDITA study)
}

Zixuan Pan ${ }^{1,2+}$, Alberto M. Marra ${ }^{3+}$, Nicola Benjamin ${ }^{1,2}$, Christina A. Eichstaedt ${ }^{1,2,4}$, Norbert Blank ${ }^{5}$, Eduardo Bossone ${ }^{6}$, Antonio Cittadini ${ }^{7}$, Gerry Coghlan ${ }^{8}$, Christopher P. Denton ${ }^{9}$, Oliver Distler ${ }^{10}$, Benjamin Egenlauf ${ }^{1,2}$, Christine Fischer $^{4}$, Satenik Harutyunova ${ }^{1,2}$, Panagiota Xanthouli, ${ }^{1,2}$, Hanns-Martin Lorenz and Ekkehard Grünig ${ }^{1,2 *}$

\begin{abstract}
Objective: The objective of this randomized, placebo-controlled, double-blind, parallel group, trial was to assess the effect of ambrisentan on mean pulmonary arterial pressure (mPAP) in patients with systemic sclerosis (SSc) and mildly elevated pulmonary hypertension (PH).

Methods: Thirty-eight SSc patients with mildly elevated mPAP at rest between 21 and $24 \mathrm{mmHg}$ and/or $>30$ $\mathrm{mmHg}$ during low-dose exercise were randomly assigned to treatment with either ambrisentan 5-10 mg/day or placebo. Right heart catheterization and further clinical parameters were assessed at baseline and after 6 months. The primary endpoint was the difference of MPAP change at rest between groups.
\end{abstract}

Results: After 6 months, the two groups did not differ in the primary endpoint (ambrisentan mPAP $-1 \pm 6.4 \mathrm{mmHg}$ vs. placebo $-0.73 \pm 3.59 \mathrm{mmHg}$ at rest, $p=0.884$ ). However, three patients from the placebo group but none of the ambrisentan group progressed to SSc-associated pulmonary arterial hypertension. Furthermore, ambrisentan treatment showed significant improvements in the secondary endpoints cardiac index $(\mathrm{Cl})$ and pulmonary vascular resistance (PVR) at rest $\left(\mathrm{Cl} 0.36 \pm 0.66 \mathrm{I} / \mathrm{min} / \mathrm{m}^{2}\right.$ vs. $-0.31 \pm 0.71 \mathrm{l} / \mathrm{min} / \mathrm{m}^{2}, p=0.010 ; \mathrm{PVR}-0.70 \pm 0.78 \mathrm{WU}$ vs. $0.01 \pm$ $0.71 \mathrm{WU}, p=0.012)$ and during exercise $\left(\mathrm{Cl} 0.7 \pm 0.81 \mathrm{l} / \mathrm{min} / \mathrm{m}^{2}\right.$ vs. $-0.45 \pm 1.36 \mathrm{l} / \mathrm{min} / \mathrm{m}^{2}, p=0.015 ; \mathrm{PVR}-0.84 \pm$ 0.48 WU vs. $-0.0032 \pm 0.34 \mathrm{WU}, p<0.0001)$.

Conclusion: This is the first randomized, double-blind, placebo-controlled study testing the effect of ambrisentan in patients with mildly elevated mPAP and/or exercise PH. The primary endpoint change in mPAP did only tendentially improve in the ambrisentan group, but the significant improvement of other hemodynamic parameters points to a possible benefit of ambrisentan and will be helpful to design future trials.

(Continued on next page)

\footnotetext{
* Correspondence: ekkehard.gruenig@med.uni-heidelberg.de

${ }^{\dagger}$ Zixuan Pan and Alberto M. Marra contributed equally to this work.

${ }^{1}$ Centre for Pulmonary Hypertension, Thoraxklinik at Heidelberg University

Hospital, Röntgenstraße 1, 69126 Heidelberg, Germany

${ }^{2}$ Translational Lung Research Center Heidelberg (TLRC), Member of the

German Center for Lung Research (DZL), Heidelberg, Germany

Full list of author information is available at the end of the article
}

(c) The Author(s). 2019 Open Access This article is distributed under the terms of the Creative Commons Attribution 4.0 International License (http://creativecommons.org/licenses/by/4.0/), which permits unrestricted use, distribution, and

reproduction in any medium, provided you give appropriate credit to the original author(s) and the source, provide a link to the Creative Commons license, and indicate if changes were made. The Creative Commons Public Domain Dedication waiver (http://creativecommons.org/publicdomain/zero/1.0/) applies to the data made available in this article, unless otherwise stated. 
(Continued from previous page)

Trial registration: www.ClinicalTrials.gov, unique identifier NCT: NCT02290613, registered $14^{\text {th }}$ of November 2014.

Keywords: Mildly elevated mPAP, Borderline pulmonary hypertension, Exercise PH, Ambrisentan, Treatment, Placebo-controlled

\section{Background}

Pulmonary hypertension (PH) is a manifestation of systemic sclerosis (SSc) [1] which dramatically impairs patients' prognosis [2]. In the American REVEAL registry, patients with SSc-associated-pulmonary arterial hypertension (PAH) (SSc-APAH) had the worst 1-, 2-, and 3year survival rates of 78,58 , and $47 \%$, respectively [3]. If left untreated, SSc-APAH patients have a median survival rate of 1 year after diagnosis [4]. Mildly elevated mean pulmonary arterial pressure (mPAP) of 21-24 $\mathrm{mmHg}$ has been shown to be associated with impaired exercise capacity and poorer outcomes when compared with individuals with mPAP within the normal range $[5-7,38]$. A recently published post hoc analysis of the DETECT study showed that SSc patients with mPAP of 21-24 $\mathrm{mmHg}$ were also more prone to develop manifest $\mathrm{PH}$ after $2.95 \pm 0.7$ years follow-up (chi-square $p$ value 0.0226) than SSc patients with mPAP of $\leq 20 \mathrm{mmHg}$ [8]. In a recent study, patients with SSc and mildly elevated mPAP presented with impaired pulmonary arterial compliance and reduced increase of cardiac index (CI) assessed by right heart catheterization (RHC) during exercise [9]. During the $6^{\text {th }}$ World Symposium on Pulmonary Hypertension, a new definition of $\mathrm{PH}$ has been proposed, lowering the cutoff for mPAP from $\geq 25$ to $>$ $20 \mathrm{mmHg}$ and including the criterion of a pulmonary vascular resistance (PVR) $>3$ Wood Units for all forms of precapillary PH [10]. This new definition may allow an earlier identification of $\mathrm{PH}$ in SSc patients.

Ambrisentan is an endothelin receptor antagonist with endothelin receptor A selectivity, which was developed for the targeted treatment of PAH. Ambrisentan was evaluated in two large phase III randomized controlled trials in ARIES (ambrisentan in pulmonary arterial hypertension, randomized, double-blind, placebo-controlled, multicenter, efficacy study)-1 and ARIES-2 for the treatment of PAH [11]. In both studies, ambrisentan has demonstrated beneficial effects on PAH symptoms, exercise capacity, hemodynamics, and time to clinical worsening, defined as occurrence of death, lung transplantation, atrial septostomy, and study withdrawal because of addition of other PAH medication or "early escape" criteria (the occurrence of two of the following: $>20 \%$ decrease of a 6 -min walking distance (6MWD), increase in the World Health Organization functional class (WHO-FC), worsening right ventricular function, hepatic or renal failure, systolic blood pressure $<85$ $\mathrm{mmHg}$ ). In PAH patients, mPAP improved about $15 \%$ due to ambrisentan [12]. Due to its beneficial effects on the development of digital ulcerations in patients with SSc $[13,14]$, the endothelin receptor antagonist bosen$\tan$ has been approved for the treatment of digital ulcers.

Up to now, apart from two small, uncontrolled openlabel reports $[15,16]$, data regarding the treatment of mildly elevated mPAP and/or exercise $\mathrm{PH}$ is lacking. As an early recognition and management of $\mathrm{PAH}$ in SSc grants a significant survival benefit [17], there is a high need of randomized, controlled studies investigating the effect of PAH-targeted treatment in SSc patients with mildly elevated mPAP and/or exercise PH. As ambrisen$\tan$ seems to have a better safety profile than the endothelin receptor antagonist bosentan with regard to hepatic safety and drug-drug interactions [18], we aimed to investigate the effect of ambrisentan on patients with SSc and mildly elevated mPAP and/or exercise PH.

The aim of this study was therefore to investigate, whether an early treatment in patients with mildly elevated mPAP and/or exercise PH may prevent these patients from worsening of hemodynamics. Mean PAP has been chosen as a primary endpoint in order to detect the progression to manifest $\mathrm{PH}$.

\section{Material and methods Study subjects}

Patients affected by diffuse cutaneous SSc (dcSSc) and limited cutaneous SSc (lcSSc) [19] referred to our center for the purpose of $\mathrm{PH}$ screening which was performed according to a modified DETECT algorithm were enrolled into the study [20]. All SSc patients received a complete non-invasive clinical assessment including echocardiography at rest and right heart catheterization at rest and during exercise. The patients were enrolled in this study if they had either (1) resting mPAP 21-24 $\mathrm{mmHg}$, pulmonary arterial wedge pressure (PAWP) < $15 \mathrm{mmHg}$, transpulmonary gradient $\quad(\mathrm{TPG}=\mathrm{mPAP}$ PAWP) $>11 \mathrm{mmHg}$, or (2) exercise-induced elevated mPAP values $>30 \mathrm{mmHg}$, PAWP $<18 \mathrm{mmHg}$, and TPG $>15 \mathrm{mmHg}$ which occurred at low workloads (cardiac output $(\mathrm{CO})<10 \mathrm{l} / \mathrm{min})$ [21] without significant left heart or severe lung disease. Inclusion of patients was based on pulmonary pressures and not on PVR, as this 
criterion [10] was not yet implemented during the conduct of the study. Left heart disease was assessed by clinical examinations including electrocardiogram (ECG), echocardiography, stress echocardiography, stress ECG, and laboratory testing of the N-terminal fraction of the pro-brain natriuretic peptide and troponin $\mathrm{T}$ (NTproBNP, TnT $\geq 14$ ). In the case of suspected coronary artery disease or any other left heart disease in patients with elevated wedge pressures, patients were referred for left heart catheterization. Lung diseases were assessed by lung function tests, by chest X-ray, and if clinically indicated by high-resolution computed tomography (HRCT). Lung involvement of SSc was considered significant when forced vital capacity (FVC) was $<60 \%$, or HRCT showed severe fibrosis, or when FVC was 60 $70 \%$ and HRCT showed moderate-severe fibrosis or was "not available," as previously described [20]. Diagnosis of SSc was confirmed by experienced rheumatologists (HML, NoB) according to the standard criteria of the American Rheumatism Association [22] with a SSc disease duration from first non-Raynaud symptoms $>3$ years. Manifest $\mathrm{PH} / \mathrm{PAH}$ was diagnosed according to the current ERS/ESC guidelines [23].

A written informed consent was obtained from all patients in accordance with the Declaration of Helsinki. The approval of the ethics committee of the Medical Faculty Heidelberg was obtained (AFmo-407/2014); the trial was registered on www.ClinicalTrials.gov (unique identifier NCT: NCT02290613) and in the European Clinical Trial Register (EudraCT Number: 2014-001882-28).

\section{Study design}

The EDITA study was a single-center (PH Center, Thoraxklinik at Heidelberg University Hospital, Heidelberg, Germany) investigator-initiated trial using a prospective, randomized, double-blind (patient and investigator), parallel group, placebo-controlled, phase IIA clinical study design. Patients were randomized 1:1 to either ambrisentan or placebo by simple randomization. Medication was prepared at the Department of Medical Pharmacy, University of Heidelberg, according to the allocation order and labeled with the patient numbers. Treatment allocation was concealed until database closure. Ambrisentan treatment was started at a dose of 5 $\mathrm{mg} /$ day and uptitrated to $10 \mathrm{mg}$ /day after $1-4$ weeks according to tolerability. Placebo tablets with the same color and shape as ambrisentan were provided by GlaxoSmithKline. Patients were assessed at baseline, after 3 and 6 months including medical history, demographics, concomitant medication, physical examination, WHOFC, vital signs, SSc characteristics, 12-lead ECG, 6MWD including Borg dyspnea score, 2-dimensional-echocardiography at rest, clinical laboratory including pregnancy test, lung function test with blood gas analysis, and monitoring of adverse events. Modified Rodnan skin score (mRSS), echocardiography, RHC with continuous 3-lead ECG at rest and during exercise, and quality of life (QoL) questionnaire (short form health survey SF36) were performed at baseline and after 6 months only. An appropriate contraception throughout the study was required for women with child-bearing potential.

\section{Endpoints}

The primary endpoint was the change from baseline to 6 months in resting mPAP with ambrisentan compared to change with placebo.

Secondary endpoints included changes from baseline to 6 months in further invasively measured hemodynamic parameters at rest and during exercise (RHC: right atrial pressure, PVR, CO, cardiac index (CI), PAWP, venous oxygen saturation $\left.\left(\mathrm{SvO}_{2}\right)\right)$, WHO-FC, mRSS and symptoms of SSc (presence of (digital) ulcers, calcinosis, dysphagia, telangiectasia, Raynaud phenomenon, and joint pain), 6MWD, QoL (SF-36), lung function tests, right heart dimensions and function assessed with transthoracic echocardiography, and NTproBNP. Measures of diseaserelated progression (adverse events, hospitalization, and initiation of PAH treatment) and the assessment of tolerability and safety were assessed throughout the study and during the safety follow-up period.

\section{Study procedures}

The hemodynamic values were obtained by RHC. The RHC was performed in a standardized way in a supine position using the transjugular access with a triplelumen 7F-Swan-Ganz thermodilution catheter at rest and during exercise as previously described [9]. Resting two-dimensional transthoracic echocardiography Doppler examinations were performed by experienced cardiac sonographers (EG, AMM, SH) with commercially available equipment (Vivid 9, GE Healthcare, Milwaukee, WI) according to a standardized protocol as described previously [24].

\section{Statistical analysis}

Statistical analyses were conducted by two statisticians (CF, NB). Data are described as mean \pm standard deviation (SD) and 95\% confidence interval or number and $\%$, respectively.

Based on data from the literature [16], we calculated that 15 patients (18 including a $20 \%$ dropout rate) would have $90 \%$ power to detect a placebo-corrected mean difference of mPAP (baseline to 6 months) of $3 \pm 2.5 \mathrm{mmHg}$ (equal standard deviation) at a two-sided significance level of 0.05 . The primary efficacy analysis was performed on data from the intention-to-treat population (all patients who received randomization) by a $t$-test 
with unequal variances (Welch tests) since the assumption for a covariance analysis was not fulfilled.

Secondary hemodynamic endpoints were tested with $t$ tests for unequal variances. Safety was analyzed descriptively. Adverse events during the study period included all adverse events that started or worsened at the time of administration of the first dose of study drug until the last visit (6 months).

Total pulmonary resistance (TPR) was calculated as mPAP-CO slope using the formula TPR $=\mathrm{mPAP}$ at peak exercise/CO at peak exercise [21]. All tests were twotailed, and $p$ values $<0.05$ were considered as statistically significant. Secondary endpoints were tested exploratory. As sensitivity analysis, changes in WHO-FC, 6-min walking test, QoL, and skin fibrosis were analyzed in patients with dcSSc only. All analyses were performed with SPSS V25 (SPSS Statistics V25, IBM Corporation, Somers, NY) and JMP14 (SAS Institute, Cary, NC).

\section{Results}

In this study, 38 SSc patients were randomly assigned to receive placebo or ambrisentan $(5-10 \mathrm{mg} /$ day) in the EDITA study from December 2014 until April 2017. Of these, 32 patients completed the study (15 in the placebo group and 17 in the ambrisentan group, Fig. 1): 78.9\% female, mean age $56.8 \pm 11.0$ years, $39.5 \%$ dcSSc, $60.5 \%$ lcSSc. All of the 38 patients had already symptoms such as shortness of breath during exercise. Baseline parameters were well balanced between the two groups (Table 1), except for SSc subtype and duration of SSc.

\section{Hemodynamics at rest and during exercise}

The primary endpoint, mean change of resting mPAP, was not significantly different between ambrisentan and placebo ( $p=0.884$, Table 2, Fig. 2). After 6 months, $\mathrm{mPAP}$ at peak exercise increased with placebo, whereas it decreased with ambrisentan, though not significantly different $(p=0.494)$.

After 6 months, 5 patients presented with resting mPAP values above $\geq 25 \mathrm{mmHg}$ (placebo $n=3$, ambrisentan $n=2$ ). The 3 patients of the placebo group were classified as manifest PAH. They were aged 25, 55, and 56 years respectively, had a resting PAWP of $\leq 15 \mathrm{mmHg}$ both at rest and during exercise at month 6 , and had shortness of breath. Their mPAP values were $24 \mathrm{mmHg}$ at baseline and increased within 6 months to values $\geq 25$ $\mathrm{mmHg}$. In all $3 \mathrm{PAH}$ patients, the disease was diagnosed at a very early stage $(27 \pm 3.5 \mathrm{mmHg})$, and in 2 patients, the increase of mPAP was only very mild (Fig. 2) most likely due to the short observation period. Up-front targeted PAH medication was started in these patients immediately after the second RHC was performed and the end of the study was reached.

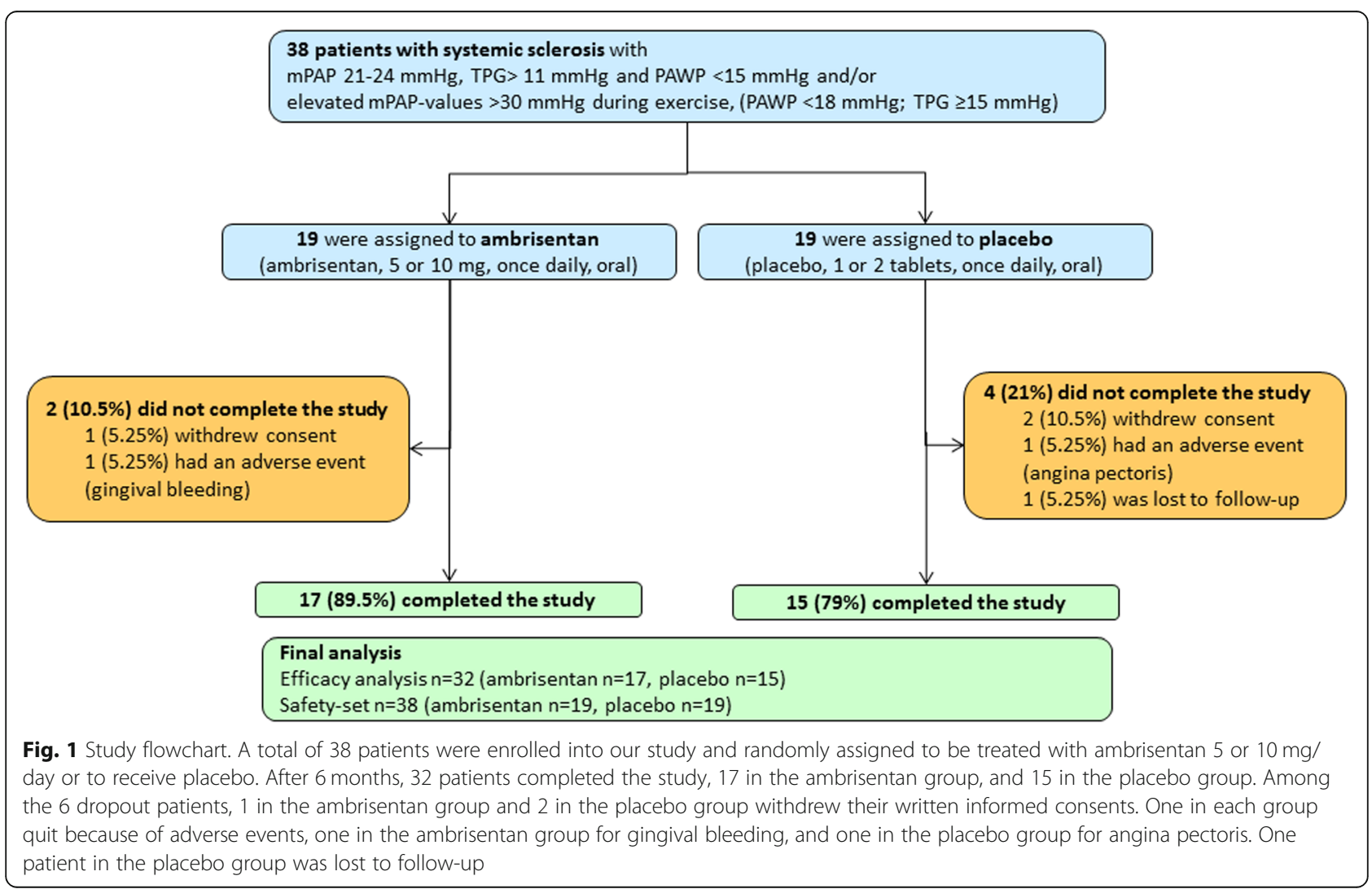


Table 1 Demographic and clinical characteristics of the study population

\begin{tabular}{|c|c|c|c|c|c|c|c|c|c|c|}
\hline \multirow[t]{2}{*}{ Parameter [unit] } & \multicolumn{3}{|l|}{$\begin{array}{l}\text { Placebo } \\
(N=19)\end{array}$} & \multicolumn{3}{|l|}{$\begin{array}{l}\text { Ambrisentan } \\
(N=19)\end{array}$} & \multirow[b]{2}{*}{$\begin{array}{l}95 \% \mathrm{Cl} \text { of } \\
\text { difference }\end{array}$} & \multicolumn{3}{|l|}{$\begin{array}{l}\text { Total } \\
(N=38)\end{array}$} \\
\hline & Mean \pm SD & $95 \% \mathrm{Cl}$ & $n$ & Mean \pm SD & $95 \% \mathrm{Cl}$ & $n$ & & Mean \pm SD & $95 \% \mathrm{Cl}$ & $n$ \\
\hline Female sex no. [\%] & $14(73.7)$ & & & $16(84.2)$ & & & & $30(78.9)$ & & \\
\hline Age [years] & $\begin{array}{l}54.89 \pm \\
11.23\end{array}$ & $\begin{array}{l}(49.48 \text { to } \\
60.31)\end{array}$ & & $\begin{array}{l}58.79 \pm \\
10.75\end{array}$ & $\begin{array}{l}(53.61 \text { to } \\
63.97)\end{array}$ & & $\begin{array}{l}(-3.33 \text { to } \\
11.12)\end{array}$ & $\begin{array}{l}56.84 \pm \\
11.02\end{array}$ & $\begin{array}{l}(53.22 \text { to } \\
60.46)\end{array}$ & \\
\hline Height $[\mathrm{cm}]$ & $\begin{array}{l}166.21 \pm \\
10.19\end{array}$ & $\begin{array}{l}(161.30 \text { to } \\
171.12)\end{array}$ & & $\begin{array}{l}166.05 \pm \\
5.59\end{array}$ & $\begin{array}{l}(163.36 \text { to } \\
168.75)\end{array}$ & & $\begin{array}{l}(-5.62 \text { to } \\
5.31)\end{array}$ & $\begin{array}{l}166.13 \pm \\
8.11\end{array}$ & $\begin{array}{l}(163.47 \text { to } \\
168.80)\end{array}$ & \\
\hline Weight [kg] & $\begin{array}{l}74.81 \pm \\
17.25\end{array}$ & $\begin{array}{l}(66.48 \text { to } \\
83.10)\end{array}$ & & $\begin{array}{l}71.45 \pm \\
15.83\end{array}$ & $\begin{array}{l}(64.03 \text { to } \\
79.22)\end{array}$ & & $\begin{array}{l}(-14.03 \text { to } \\
7.70)\end{array}$ & $\begin{array}{l}73.13 \pm \\
16.42\end{array}$ & $\begin{array}{l}(67.83 \text { to } \\
78.59)\end{array}$ & \\
\hline Systolic blood pressure $[\mathrm{mmHg}]$ & $\begin{array}{l}121.32 \pm \\
14.99\end{array}$ & $\begin{array}{l}(114.09 \text { to } \\
128.54)\end{array}$ & & $\begin{array}{l}116.32 \pm \\
11.53\end{array}$ & $\begin{array}{l}(110.76 \text { to } \\
121.87)\end{array}$ & & $\begin{array}{l}(-13.80 \text { to } \\
3.80)\end{array}$ & $\begin{array}{l}118.82 \pm \\
13.43\end{array}$ & $\begin{array}{l}(114.40 \text { to } \\
123.23)\end{array}$ & \\
\hline Diastolic blood pressure $[\mathrm{mmHg}]$ & $73.42 \pm 9.14$ & $\begin{array}{l}(69.02 \text { to } \\
77.82)\end{array}$ & & $73.68 \pm 8.95$ & $\begin{array}{l}(69.37 \text { to } \\
78.00)\end{array}$ & & $\begin{array}{l}(-5.69 \text { to } \\
6.21)\end{array}$ & $73.55 \pm 8.92$ & $\begin{array}{l}\text { (70.62 to } \\
76.49)\end{array}$ & \\
\hline HR [beats/min] & $77.58 \pm 9.85$ & $\begin{array}{l}\text { (72.83 to } \\
82.33)\end{array}$ & & $\begin{array}{l}73.05 \pm \\
11.12\end{array}$ & $\begin{array}{l}(67.69 \text { to } \\
78.41)\end{array}$ & & $\begin{array}{l}(-11.44 \text { to } \\
2.39)\end{array}$ & $\begin{array}{l}75.32 \pm \\
10.61\end{array}$ & $\begin{array}{l}\text { (72.83 to } \\
78.81)\end{array}$ & \\
\hline \multicolumn{11}{|l|}{ WHO-FC no. [\%] } \\
\hline$\|$ & $15(78.9)$ & & & $17(89.5)$ & & & & $32(84.2)$ & & \\
\hline III & $4(21.1)$ & & & $2(10.5)$ & & & & $6(15.8)$ & & \\
\hline \multicolumn{11}{|c|}{ Hemodynamic characteristic according to inclusion criteria } \\
\hline $\begin{array}{l}\mathrm{mPAP} \text { rest } 21-24 \mathrm{mmHg}, \mathrm{mPAP} \\
\text { exercise }>30 \mathrm{mmHg}\end{array}$ & $12(63.2)$ & & & $10(52.6)$ & & & & $22(57.9)$ & & \\
\hline $\begin{array}{l}\mathrm{mPAP} \text { rest } 21-24 \mathrm{mmHg}, \mathrm{mPAP} \\
\text { exercise } \leq 30 \mathrm{mmHg}\end{array}$ & $3(15.8)$ & & & $1(5.3)$ & & & & $4(10.5)$ & & \\
\hline $\begin{array}{l}\text { mPAP rest }<21 \mathrm{mmHg} \text {, mPAP } \\
\text { exercise }>30 \mathrm{mmHg}\end{array}$ & $4(21.0)$ & & & $8(42.1)$ & & & & $2(31.6)$ & & \\
\hline mRSS [points] & $11.47 \pm 5.22$ & $\begin{array}{l}(8.72 \text { to } \\
14.23)\end{array}$ & & $11.47 \pm 5.71$ & $\begin{array}{l}(8.96 \text { to } \\
13.99)\end{array}$ & & $\begin{array}{l}(-3.60 \text { to } \\
3.60)\end{array}$ & $11.47 \pm 5.40$ & $\begin{array}{l}(9.70 \text { to } \\
13.25)\end{array}$ & \\
\hline \multicolumn{11}{|l|}{ SSc subgroup, no. (\%) } \\
\hline Diffuse & $11(57.9)$ & & & $4(21.1)$ & & & & 15 (39.5) & & \\
\hline Limited & $8(42.1)$ & & & $15(78.9)$ & & & & $23(60.5)$ & & \\
\hline SSC disease duration [years] & $\begin{array}{l}13.28 \pm \\
10.85\end{array}$ & $\begin{array}{l}\text { (8.05 to } \\
18.51)\end{array}$ & & $6.63 \pm 4.80$ & (4.32 to 8.96$)$ & & $\begin{array}{l}(-12.17 \text { to } \\
1.12)\end{array}$ & $9.96 \pm 8.93$ & $\begin{array}{l}(7.02 \text { to } \\
12.90)\end{array}$ & \\
\hline \multicolumn{11}{|l|}{ Hemodynamics at rest } \\
\hline $\mathrm{CVP}[\mathrm{mmHg}]$ & $5.63 \pm 2.97$ & (4.20 to 7.06$)$ & & $6.11 \pm 2.94$ & (4.69 to 7.52 ) & & $\begin{array}{l}(-1.47 \text { to } \\
2.41)\end{array}$ & $5.87 \pm 2.92$ & (4.90 to 6.82$)$ & \\
\hline $\mathrm{mPAP}[\mathrm{mmHg}]$ & $21.32 \pm 2.43$ & $\begin{array}{l}(20.15 \\
\text { to22.49) }\end{array}$ & & $19.84 \pm 3.58$ & $\begin{array}{l}(18.12 \text { to } \\
21.57)\end{array}$ & & $\begin{array}{l}(-3.49 \text { to } \\
0.54)\end{array}$ & $20.58 \pm 3.11$ & $\begin{array}{l}(19.56 \text { to } \\
21.60)\end{array}$ & \\
\hline PAWP $[\mathrm{mmHg}]$ & $9.58 \pm 2.97$ & $\begin{array}{l}\text { (8.15 to } \\
11.01)\end{array}$ & & $9.42 \pm 2.32$ & $\begin{array}{l}\text { (8.30 to } \\
10.54)\end{array}$ & & $\begin{array}{l}(-1.91 \text { to } \\
1.59)\end{array}$ & $9.50 \pm 2.63$ & $\begin{array}{l}(8.64 \text { to } \\
10.37)\end{array}$ & \\
\hline $\mathrm{CO}[\mathrm{l} / \mathrm{min}]$ & $5.66 \pm 1.48$ & (4.95 to 6.37 ) & & $5.04 \pm 1.26$ & (4.43 to 5.65 ) & & $\begin{array}{l}(-1.51 \text { to } \\
0.29)\end{array}$ & $5.35 \pm 1.39$ & (4.89 to 5.81 ) & \\
\hline $\mathrm{Cl}\left[1 / \mathrm{min} / \mathrm{m}^{2}\right]$ & $3.20 \pm 0.85$ & (2.79 to 3.61 ) & & $2.84 \pm 0.64$ & (2.53 to 3.14$)$ & & $\begin{array}{l}(-0.86 \text { to } \\
0.13)\end{array}$ & $3.02 \pm 0.76$ & (2.77 to 3.27 ) & \\
\hline $\mathrm{SvO}_{2}[\%]$ & $73.13 \pm 4.60$ & $\begin{array}{l}\text { (70.67 to } \\
75.58)\end{array}$ & (16) & $73.91 \pm 8.58$ & $\begin{array}{l}(69.50 \text { to } \\
78.32)\end{array}$ & (17) & $\begin{array}{l}(-4.15 \text { to } \\
5.71)\end{array}$ & $73.53 \pm 6.85$ & $\begin{array}{l}\text { (71.10 to } \\
75.96)\end{array}$ & (33) \\
\hline PVR [WU] & $2.09 \pm 0.61$ & (1.80 to 2.39$)$ & & $2.22 \pm 0.93$ & (1.77 to 2.67$)$ & & $\begin{array}{l}(-0.39 \text { to } \\
0.65)\end{array}$ & $2.16 \pm 0.78$ & (1.90 to 2.41$)$ & \\
\hline \multicolumn{11}{|l|}{ Hemodynamics at peak exercise } \\
\hline $\mathrm{mPAP}[\mathrm{mmHg}]$ & $36.94 \pm 6.08$ & $\begin{array}{l}\text { (33.82 to } \\
40.07)\end{array}$ & (17) & $37.78 \pm 3.61$ & $\begin{array}{l}\text { (35.98 to } \\
39.57)\end{array}$ & (18) & $\begin{array}{l}(-2.58 \text { to } \\
2.25)\end{array}$ & $37.37 \pm 4.91$ & $\begin{array}{l}\text { (35.69 to } \\
39.06)\end{array}$ & (35) \\
\hline PAWP [mmHg] & $15.24 \pm 5.43$ & $\begin{array}{l}\text { (12.45 to } \\
18.03)\end{array}$ & (17) & $16.78 \pm 6.42$ & $\begin{array}{l}(13.58 \text { to } \\
19.97)\end{array}$ & (18) & $\begin{array}{l}(-2.56 \text { to } \\
5.64)\end{array}$ & $16.03 \pm 5.92$ & $\begin{array}{l}\text { (13.99 to } \\
18.06)\end{array}$ & (35) \\
\hline $\mathrm{CO}[\mathrm{l} / \mathrm{min}]$ & $11.03 \pm 3.62$ & (9.17 to & (17) & $9.67 \pm 2.67$ & (8.34 to & (18) & $(-3.54$ to & $10.33 \pm 3.19$ & (9.23 to & (35) \\
\hline
\end{tabular}


Table 1 Demographic and clinical characteristics of the study population (Continued)

\begin{tabular}{|c|c|c|c|c|c|c|c|c|c|c|}
\hline \multirow[t]{2}{*}{ Parameter [unit] } & \multicolumn{3}{|l|}{$\begin{array}{l}\text { Placebo } \\
(N=19)\end{array}$} & \multicolumn{3}{|l|}{$\begin{array}{l}\text { Ambrisentan } \\
(N=19)\end{array}$} & \multirow[b]{2}{*}{$\begin{array}{l}95 \% \mathrm{Cl} \text { of } \\
\text { difference }\end{array}$} & \multicolumn{3}{|l|}{$\begin{array}{l}\text { Total } \\
(N=38)\end{array}$} \\
\hline & Mean \pm SD & $95 \% \mathrm{Cl}$ & $n$ & Mean \pm SD & $95 \% \mathrm{Cl}$ & $n$ & & Mean \pm SD & $95 \% \mathrm{Cl}$ & $n$ \\
\hline & & 12.89) & & & 10.99) & & $0.81)$ & & 11.43) & \\
\hline $\mathrm{Cl}\left[\mathrm{l} / \mathrm{min} / \mathrm{m}^{2}\right]$ & $6.04 \pm 1.79$ & (5.12 to 6.96$)$ & (17) & $5.41 \pm 1.32$ & (4.75 to 6.06$)$ & (18) & $\begin{array}{l}(-1.71 \text { to } \\
0.45)\end{array}$ & $5.71 \pm 1.58$ & (5.17 to 6.25 ) & $(35$ \\
\hline $\mathrm{SvO}_{2}[\%]$ & $38.27 \pm 6.10$ & $\begin{array}{l}\text { (34.89 to } \\
41.64)\end{array}$ & (15) & $\begin{array}{l}42.77 \pm \\
11.56\end{array}$ & $\begin{array}{l}\text { (36.36 to } \\
49.17)\end{array}$ & (15) & $\begin{array}{l}(-2.42 \text { to } \\
11.42)\end{array}$ & $40.52 \pm 9.37$ & $\begin{array}{l}\text { (37.02 to } \\
44.02 \text { ) }\end{array}$ & $(30$ \\
\hline Workload [Watt] & $\begin{array}{l}75.00 \pm \\
34.23\end{array}$ & $\begin{array}{l}(57.40 \text { to } \\
92.60)\end{array}$ & (17) & $\begin{array}{l}75.00 \pm \\
27.12\end{array}$ & $\begin{array}{l}(61.52 \text { to } \\
88.48)\end{array}$ & (18) & $\begin{array}{l}(-21.18 \text { to } \\
21.17)\end{array}$ & $\begin{array}{l}75.00 \pm \\
30.32\end{array}$ & $\begin{array}{l}(65.59 \text { to } \\
85.41)\end{array}$ & $(35$ \\
\hline $\mathrm{HR} \max [/ \mathrm{min}]$ & $\begin{array}{l}117.65 \pm \\
21.37\end{array}$ & $\begin{array}{l}\text { (106.66 to } \\
128.63)\end{array}$ & (17) & $\begin{array}{l}111.39 \pm \\
23.06\end{array}$ & $\begin{array}{l}\text { (99.92 to } \\
122.86)\end{array}$ & (18) & $\begin{array}{l}(-21.57 \text { to } \\
9.06)\end{array}$ & $\begin{array}{l}114.43 \pm \\
22.15\end{array}$ & $\begin{array}{l}(106.82 \text { to } \\
122.04)\end{array}$ & (35) \\
\hline PVR [WU] & $2.07 \pm 0.61$ & (1.76 to 2.38$)$ & (17) & $2.29 \pm 0.85$ & (1.87 to 2.71$)$ & (18) & $\begin{array}{l}(-0.29 \text { to } \\
0.73)\end{array}$ & $2.18 \pm 0.74$ & (1.93 to 2.44$)$ & (35) \\
\hline TPR $\left[\mathrm{mmHg} \min \mathrm{I}^{-1}\right]$ & $3.65 \pm 1.18$ & (3.04 to 4.26 ) & (17) & $4.28 \pm 1.65$ & (3.46 to 5.10$)$ & (18) & $\begin{array}{l}(-0.36 \text { to } \\
1.62)\end{array}$ & $3.97 \pm 1.46$ & (3.47 to 4.47 ) & (35) \\
\hline \multicolumn{11}{|l|}{ 6MWD } \\
\hline $6 \mathrm{MWD}[\mathrm{m}]$ & $\begin{array}{l}448.11 \pm \\
82.64\end{array}$ & $\begin{array}{l}(408.28 \text { to } \\
487.93)\end{array}$ & & $\begin{array}{l}470.21 \pm \\
77.03\end{array}$ & $\begin{array}{l}(433.08 \text { to } \\
407.34)\end{array}$ & & $\begin{array}{l}(-30.46 \text { to } \\
74.67)\end{array}$ & $\begin{array}{l}459.16 \pm \\
79.59\end{array}$ & $\begin{array}{l}(433.00 \text { to } \\
485.32)\end{array}$ & \\
\hline Borg dyspnea score & $2.93 \pm 1.97$ & (1.98 to 3.88$)$ & & $2.41 \pm 1.32$ & (1.77 to 3.04$)$ & & $\begin{array}{l}(-1.63 \text { to } \\
0.58)\end{array}$ & $2.67 \pm 1.68$ & (2.11 to 3.22 ) & \\
\hline $\mathrm{SaO}_{2}$ after $6 \mathrm{MWD}[\%]$ & $91.87 \pm 4.63$ & $\begin{array}{l}(89.30 \text { to } \\
94.43)\end{array}$ & (15) & $91.71 \pm 4.79$ & $\begin{array}{l}\text { (89.24 to } \\
94.17)\end{array}$ & (17) & $\begin{array}{l}(-3.57 \text { to } \\
3.25)\end{array}$ & $91.78 \pm 4.64$ & $\begin{array}{l}\text { (90.10 to } \\
93.45 \text { ) }\end{array}$ & $(32)$ \\
\hline HR after 6MWD [/min] & $99 \pm 21.27$ & $\begin{array}{l}\text { (88.07 to } \\
109.93 \text { ) }\end{array}$ & (17) & $107 \pm 19.64$ & $\begin{array}{l}\text { (96.90 to } \\
117.10)\end{array}$ & (17) & $\begin{array}{l}(-6.30 \text { to } \\
22.30)\end{array}$ & $103 \pm 20.56$ & $\begin{array}{l}\text { (95.82 to } \\
110.17 \text { ) }\end{array}$ & (34) \\
\hline \multicolumn{10}{|l|}{ Echocardiography at rest } & \\
\hline Estimated sPAP [mmHg] & $29.21 \pm 5.16$ & $\begin{array}{l}\text { (25.25 to } \\
31.17)\end{array}$ & & $28.58 \pm 6.57$ & $\begin{array}{l}\text { (26.04 to } \\
31.43 \text { ) }\end{array}$ & & $\begin{array}{l}(-4.52 \text { to } \\
3.26)\end{array}$ & $28.89 \pm 5.83$ & $\begin{array}{l}\text { (26.98 to } \\
30.81 \text { ) }\end{array}$ & \\
\hline RA area $\left[\mathrm{cm}^{2}\right]$ & $12.05 \pm 4.24$ & $\begin{array}{l}(10.73 \text { to } \\
13.69)\end{array}$ & & $11.68 \pm 3.28$ & $\begin{array}{l}(10.45 \text { to } \\
13.66)\end{array}$ & & $\begin{array}{l}(-2.86 \text { to } \\
2.13)\end{array}$ & $11.87 \pm 3.74$ & $\begin{array}{l}(10.64 \text { to } \\
13.10)\end{array}$ & \\
\hline $\mathrm{RV}$ area $\left[\mathrm{cm}^{2}\right]$ & $14.89 \pm 4.56$ & $\begin{array}{l}\text { (12.97 to } \\
17.03)\end{array}$ & & $14.13 \pm 4.74$ & $\begin{array}{l}(12.65 \text { to } \\
16.82)\end{array}$ & & $\begin{array}{l}(-3.82 \text { to } \\
2.30)\end{array}$ & $14.51 \pm 4.60$ & $(13.00-16.03)$ & \\
\hline TAPSE $[\mathrm{cm}]$ & $2.50 \pm 0.51$ & (2.23 to 2.71$)$ & & $2.41 \pm 0.33$ & (2.28 to 2.70$)$ & & $\begin{array}{l}(-0.37 \text { to } \\
0.20)\end{array}$ & $\begin{array}{l}16,834 \pm \\
0.43\end{array}$ & (2.31 to 2.60 ) & \\
\hline \multicolumn{10}{|l|}{ Lung function } & \\
\hline FVC [\%] & $\begin{array}{l}69.17 \pm \\
13.72\end{array}$ & $\begin{array}{l}(62.56 \text { to } \\
75.78)\end{array}$ & & $\begin{array}{l}70.51 \pm \\
17.61\end{array}$ & $\begin{array}{l}(62.02 \text { to } \\
79.00)\end{array}$ & & $\begin{array}{l}(-6.37 \text { to } \\
1.84)\end{array}$ & $\begin{array}{l}69.84 \pm \\
15.58\end{array}$ & $\begin{array}{l}(64.72 \text { to } \\
74.96)\end{array}$ & \\
\hline FEV1 [L] & $2.40 \pm 0.77$ & (2.03 to 2.77 ) & & $2.26 \pm 0.63$ & (1.96 to 2.57$)$ & & $\begin{array}{l}(-0.60 \text { to } \\
0.33)\end{array}$ & $2.33 \pm 0.70$ & (2.10 to 2.56$)$ & \\
\hline FEV1\% VC max [\%] & $79.31 \pm 6.98$ & $\begin{array}{l}\text { (75.95 to } \\
82.68)\end{array}$ & & $\begin{array}{l}81.44 \pm \\
15.38\end{array}$ & $\begin{array}{l}\text { (74.03 to } \\
88.85 \text { ) }\end{array}$ & & $\begin{array}{l}(-5.73 \text { to } \\
9.99)\end{array}$ & $\begin{array}{l}80.38 \pm \\
11.83\end{array}$ & $\begin{array}{l}(76.49 \text { to } \\
84.27)\end{array}$ & \\
\hline PEF $[1 / s]$ & $5.46 \pm 2.32$ & (4.34 to 6.58$)$ & & $5.17 \pm 1.72$ & (4.34 to 6.00$)$ & & $\begin{array}{l}(-1.63 \text { to } \\
1.06)\end{array}$ & $5.31 \pm 2.02$ & (4.65 to 5.98 ) & \\
\hline TLC [l] & $4.96 \pm 1.07$ & (4.44 to 5.48 ) & & $5.08 \pm 1.25$ & (4.48 to 5.69$)$ & & $\begin{array}{l}(-0.64 \text { to } \\
0.89)\end{array}$ & $5.02 \pm 1.15$ & (4.64 to 5.40 ) & \\
\hline Residual volume [l] & $1.94 \pm 0.55$ & (1.68 to 2.21$)$ & & $2.18 \pm 0.70$ & (1.84 to 2.52$)$ & & $\begin{array}{l}(-0.18 \text { to } \\
0.65)\end{array}$ & $2.06 \pm 0.63$ & (1.85 to 2.27$)$ & \\
\hline $\mathrm{DLCO}[\mathrm{mmol} / \mathrm{min} / \mathrm{kPa}]$ & $5.28 \pm 1.52$ & (4.55 to 6.02$)$ & & $5.03 \pm 1.33$ & (4.38 to 5.67 ) & & $\begin{array}{l}(-1.20 \text { to } \\
0.68)\end{array}$ & $5.16 \pm 1.42$ & (4.69 to 5.62 ) & \\
\hline DLCO \% predicted & $84.73 \pm 2.61$ & $\begin{array}{l}(83.47 \text { to } \\
85.99)\end{array}$ & & $84.16 \pm 1.89$ & $\begin{array}{l}\text { (83.25 to } \\
85.08)\end{array}$ & & $\begin{array}{l}(-2.07 \text { to } \\
0.93)\end{array}$ & $84.45 \pm 2.27$ & $\begin{array}{l}\text { (83.70 to } \\
85.19)\end{array}$ & \\
\hline $\mathrm{SaO}_{2}[\%]$ & $96.13 \pm 1.80$ & $\begin{array}{l}\text { (95.26 to } \\
97.00)\end{array}$ & & $96.82 \pm 0.77$ & $\begin{array}{l}\text { (96.45 to } \\
97.17)\end{array}$ & & $\begin{array}{l}(-0.23 \text { to } \\
1.60)\end{array}$ & $96.47 \pm 1.41$ & $\begin{array}{l}\text { (96.01 to } \\
96.94)\end{array}$ & \\
\hline $\mathrm{PaO}_{2}[\mathrm{mmHg}]$ & $78.78 \pm 9.42$ & $\begin{array}{l}\text { (74.24 to } \\
83.32 \text { ) }\end{array}$ & & $81.22 \pm 5.91$ & $\begin{array}{l}\text { (78.37 to } \\
84.06)\end{array}$ & & $\begin{array}{l}(-2.74 \text { to } \\
7.61)\end{array}$ & $80.00 \pm 7.85$ & $\begin{array}{l}\text { (77.42 to } \\
82.58 \text { ) }\end{array}$ & \\
\hline
\end{tabular}


Table 1 Demographic and clinical characteristics of the study population (Continued)

\begin{tabular}{|c|c|c|c|c|c|c|c|c|c|c|}
\hline \multirow[t]{2}{*}{ Parameter [unit] } & \multicolumn{3}{|l|}{$\begin{array}{l}\text { Placebo } \\
(N=19)\end{array}$} & \multicolumn{3}{|l|}{$\begin{array}{l}\text { Ambrisentan } \\
(N=19)\end{array}$} & \multirow[b]{2}{*}{$\begin{array}{l}95 \% \mathrm{Cl} \text { of } \\
\text { difference }\end{array}$} & \multicolumn{3}{|l|}{$\begin{array}{l}\text { Total } \\
(N=38)\end{array}$} \\
\hline & Mean \pm SD & $95 \% \mathrm{Cl}$ & $n$ & Mean \pm SD & $95 \% \mathrm{Cl}$ & $n$ & & Mean \pm SD & $95 \% \mathrm{Cl}$ & $n$ \\
\hline $\mathrm{PaCO}_{2}[\mathrm{mmHg}]$ & $39.01 \pm 3.63$ & $\begin{array}{l}(37.25 \text { to } \\
40.76)\end{array}$ & & $37.23 \pm 2.57$ & $\begin{array}{l}\text { (35.99 to } \\
38.47 \text { ) }\end{array}$ & & $\begin{array}{l}(-3.85 \text { to } \\
0.29)\end{array}$ & $38.12 \pm 3.23$ & $\begin{array}{l}\text { (37.05 to } \\
39.18)\end{array}$ & \\
\hline \multicolumn{11}{|l|}{ Laboratory } \\
\hline Hemoglobin $[\mathrm{g} / \mathrm{dl}]$ & $13.54 \pm 1.26$ & $\begin{array}{l}(12.93 \text { to } \\
14.14)\end{array}$ & & $13.62 \pm 1.13$ & $\begin{array}{l}\text { (13.07 to } \\
14.16)\end{array}$ & & $\begin{array}{l}(-0.71 \text { to } \\
0.87)\end{array}$ & $13.58 \pm 1.18$ & $\begin{array}{l}\text { (13.19 to } \\
13.96)\end{array}$ & \\
\hline Hematocrit [l/l] & $0.42 \pm 0.03$ & (0.40 to 0.43 ) & & $0.41 \pm 0.03$ & (0.40 to 0.43 ) & & $\begin{array}{l}(-0.03 \text { to } \\
0.02)\end{array}$ & $0.41 \pm 0.03$ & (0.40 to 0.42$)$ & \\
\hline Platelet [100/nl] & $2.53 \pm 0.94$ & (2.08 to 2.98 ) & & $2.62 \pm 0.63$ & (2.31 to 2.92 ) & & $\begin{array}{l}(-0.44 \text { to } \\
0.61)\end{array}$ & $2.58 \pm 0.79$ & (2.32 to 2.83 ) & \\
\hline Creatinine $[\mathrm{mg} / \mathrm{dl}]$ & $0.83 \pm 0.16$ & (0.75 to 0.91$)$ & & $0.86 \pm 0.12$ & (0.80 to 0.92$)$ & & $\begin{array}{l}(-0.07 \text { to } \\
0.13)\end{array}$ & $0.85 \pm 0.14$ & (0.80 to 0.89 ) & \\
\hline Potassium [mmol/l] & $4.05 \pm 0.38$ & (3.86 to 4.23 ) & & $4.20 \pm 0.48$ & (3.97 to 4.43 ) & & $\begin{array}{l}(-0.13 \text { to } \\
0.44)\end{array}$ & $4.12 \pm 0.43$ & (3.98 to 4.27 ) & \\
\hline AST [U/I] & $\begin{array}{l}24.11 \pm \\
21.78\end{array}$ & $\begin{array}{l}(13.61 \text { to } \\
34.60)\end{array}$ & & $19.79 \pm 7.06$ & $\begin{array}{l}\text { (16.39 to } \\
23.19)\end{array}$ & & $\begin{array}{l}(-14.97 \text { to } \\
6.34)\end{array}$ & $\begin{array}{l}21.95 \pm \\
16.12\end{array}$ & $\begin{array}{l}(16.65 \text { to } \\
27.25)\end{array}$ & \\
\hline $\mathrm{ALT}[\mathrm{U} / \mathrm{I}]$ & $\begin{array}{l}29.68 \pm \\
24.93\end{array}$ & $\begin{array}{l}(17.67 \text { to } \\
41.70)\end{array}$ & & $24.68 \pm 9.85$ & $\begin{array}{l}\text { (19.93 to } \\
29.43)\end{array}$ & & $\begin{array}{l}(-17.47 \text { to } \\
7.47)\end{array}$ & $\begin{array}{l}27.18 \pm \\
18.87\end{array}$ & $\begin{array}{l}\text { (20.98 to } \\
33.39)\end{array}$ & \\
\hline $\mathrm{LDH}[\mathrm{U} / \mathrm{I}]$ & $\begin{array}{l}197.63 \pm \\
54.74\end{array}$ & $\begin{array}{l}(171.25 \text { to } \\
224.01)\end{array}$ & & $\begin{array}{l}197.53 \pm \\
35.59\end{array}$ & $\begin{array}{l}(180.37 \text { to } \\
214.68)\end{array}$ & & $\begin{array}{l}(-30.48 \text { to } \\
30.27)\end{array}$ & $\begin{array}{l}197.58 \pm \\
45.54\end{array}$ & $\begin{array}{l}\text { (182.61 to } \\
212.55)\end{array}$ & \\
\hline $\mathrm{CRP}[\mathrm{mg} / \mathrm{l}]$ & $5.18 \pm 5.17$ & (2.69 to 7.68 ) & & $5.28 \pm 11.60$ & $\begin{array}{l}(-0.48 \text { to } \\
11.05)\end{array}$ & (18) & $\begin{array}{l}(-5.84 \text { to } \\
6.04)\end{array}$ & $5.23 \pm 8.77$ & (2.30 to 8.16$)$ & (37) \\
\hline NTproBNP [pg/ml] & $\begin{array}{l}123.42 \pm \\
142.96\end{array}$ & $\begin{array}{l}(54.52 \text { to } \\
192.32)\end{array}$ & & $\begin{array}{l}267.83 \pm \\
303.11\end{array}$ & $\begin{array}{l}\text { (117.10 to } \\
418.57)\end{array}$ & (18) & $\begin{array}{l}(-12.38 \text { to } \\
301.20)\end{array}$ & $\begin{array}{l}193.68 \pm \\
242.81\end{array}$ & $\begin{array}{l}(112.71 \text { to } \\
274.63)\end{array}$ & (37) \\
\hline \multicolumn{11}{|l|}{ Quality of life SF-36 } \\
\hline Physical functioning & $\begin{array}{l}50.26 \pm \\
25.95\end{array}$ & $\begin{array}{l}(37.75 \text { to } \\
62.77)\end{array}$ & & $\begin{array}{l}64.21 \pm \\
25.83\end{array}$ & $\begin{array}{l}\text { (51.67 to } \\
76.66)\end{array}$ & & $\begin{array}{l}(-3.09 \text { to } \\
30.99)\end{array}$ & $\begin{array}{l}57.24 \pm \\
26.50\end{array}$ & $\begin{array}{l}(48.52 \text { to } \\
65.95)\end{array}$ & \\
\hline Physical role functioning & $\begin{array}{l}35.53 \pm \\
40.24\end{array}$ & $\begin{array}{l}(16.13 \text { to } \\
54.92)\end{array}$ & & $\begin{array}{l}51.32 \pm \\
41.23\end{array}$ & $\begin{array}{l}\text { (31.45 to } \\
79.19)\end{array}$ & & $\begin{array}{l}(-11.01 \text { to } \\
42.59)\end{array}$ & $\begin{array}{l}43.42 \pm \\
40.97\end{array}$ & $\begin{array}{l}\text { (29.96 to } \\
56.89)\end{array}$ & \\
\hline Bodily pain & $\begin{array}{l}49.79 \pm \\
28.96\end{array}$ & $\begin{array}{l}\text { (35.83 to } \\
63.75)\end{array}$ & & $\begin{array}{l}62.00 \pm \\
29.16\end{array}$ & $\begin{array}{l}\text { (47.95 to } \\
76.05)\end{array}$ & & $\begin{array}{l}(-6.91 \text { to } \\
31.33)\end{array}$ & $\begin{array}{l}55.89 \pm \\
29.33\end{array}$ & $\begin{array}{l}\text { (46.26 to } \\
65.53)\end{array}$ & \\
\hline General health perceptions & $\begin{array}{l}41.74 \pm \\
13.07\end{array}$ & $\begin{array}{l}\text { (35.44 to } \\
48.04 \text { ) }\end{array}$ & & $\begin{array}{l}54.42 \pm \\
19.47\end{array}$ & $\begin{array}{l}\text { (45.03 to } \\
63.81)\end{array}$ & & (1.72 to 23.65$)$ & $\begin{array}{l}48.08 \pm \\
17.58\end{array}$ & $\begin{array}{l}\text { (42.30 to } \\
53.86)\end{array}$ & \\
\hline Vitality & $\begin{array}{l}42.89 \pm \\
19.32\end{array}$ & $\begin{array}{l}(33.58 \text { to } \\
52.20)\end{array}$ & & $\begin{array}{l}50.53 \pm \\
20.94\end{array}$ & $\begin{array}{l}\text { (40.03 to } \\
60.62)\end{array}$ & & $\begin{array}{l}(-5.62 \text { to } \\
20.89)\end{array}$ & $\begin{array}{l}46.71 \pm \\
20.24\end{array}$ & $\begin{array}{l}\text { (40.06 to } \\
53.37)\end{array}$ & \\
\hline Social role functioning & $\begin{array}{l}58.05 \pm \\
25.99\end{array}$ & $\begin{array}{l}\text { ( } 45.53 \text { to } \\
70.58)\end{array}$ & & $\begin{array}{l}74.47 \pm \\
24.45\end{array}$ & $\begin{array}{l}\text { (62.69 to } \\
86.26)\end{array}$ & & $\begin{array}{l}(-0.18 \text { to } \\
33.03)\end{array}$ & $\begin{array}{l}66.26 \pm \\
26.24\end{array}$ & $\begin{array}{l}\text { (57.64 to } \\
74.89)\end{array}$ & \\
\hline Emotional role functioning & $\begin{array}{l}49.16 \pm \\
46.33\end{array}$ & $\begin{array}{l}\text { (26.83 to } \\
71.49)\end{array}$ & & $\begin{array}{l}63.16 \pm \\
47.02\end{array}$ & $\begin{array}{l}\text { ( } 40.50 \text { to } \\
85.82 \text { ) }\end{array}$ & & $\begin{array}{l}(-16.71 \text { to } \\
44.71)\end{array}$ & $\begin{array}{l}56.16 \pm \\
46.58\end{array}$ & $\begin{array}{l}\text { (40.85 to } \\
71.47)\end{array}$ & \\
\hline Mental health & $\begin{array}{l}59.58 \pm \\
18.85\end{array}$ & $\begin{array}{l}(50.49 \text { to } \\
68.66)\end{array}$ & & $\begin{array}{l}64.42 \pm \\
18.03\end{array}$ & $\begin{array}{l}(55.73 \text { to } \\
73.11)\end{array}$ & & $\begin{array}{l}(-7.30 \text { to } \\
16.98)\end{array}$ & $\begin{array}{l}62.00 \pm \\
18.36\end{array}$ & $\begin{array}{l}\text { (55.97 to } \\
68.04)\end{array}$ & \\
\hline Physical health score & $\begin{array}{l}43.89 \pm \\
21.26\end{array}$ & $\begin{array}{l}\text { (33.65 to } \\
54.14)\end{array}$ & & $\begin{array}{l}56.47 \pm \\
23.87\end{array}$ & $\begin{array}{l}\text { (44.97 to } \\
67.98)\end{array}$ & & $\begin{array}{l}(-2.29 \text { to } \\
27.45)\end{array}$ & $\begin{array}{l}50.18 \pm \\
23.19\end{array}$ & $\begin{array}{l}(42.56 \text { to } \\
57.81)\end{array}$ & \\
\hline Mental health score & $\begin{array}{l}50.26 \pm \\
20.70\end{array}$ & $\begin{array}{l}(40.29 \text { to } \\
60.24)\end{array}$ & & $\begin{array}{l}61.42 \pm \\
21.45\end{array}$ & $\begin{array}{l}(51.08 \text { to } \\
71.76)\end{array}$ & & $\begin{array}{l}(-2.71 \text { to } \\
25.03)\end{array}$ & $\begin{array}{l}55.84 \pm \\
21.55\end{array}$ & $\begin{array}{l}(48.76 \text { to } \\
62.92)\end{array}$ & \\
\hline
\end{tabular}

$S D$ standard deviation, $H R$ heart rate, $m R S S$ modified Rodnan Skin Score, WHO-FC World Health Organization functional class, CVP central venous pressure, mPAP mean pulmonary arterial pressure, $P A W P$ pulmonary capillary wedge pressure, $\mathrm{CO}$ cardiac output, $\mathrm{Cl}$ cardiac index, $\mathrm{SvO} \mathrm{O}_{2}$ venous oxygen saturation, $P V R$ pulmonary vascular resistance, WU Wood Units, sPAP systolic pulmonary arterial pressure, $R A$ right atrial, $R V$ right ventricular, TAPSE tricuspid annular plane systolic excursion, FVC forced vital capacity, FEV1 forced expiratory volume in first second, VC vital capacity, PEF peak expiratory flow, $T L C$ total lung capacity, $\mathrm{DLCO}$ diffusing capacity of the lung for carbon monoxide, $\mathrm{SaO}_{2}$ oxygen saturation, $\mathrm{PaO} \mathrm{O}_{2}$ partial pressure of oxygen, $\mathrm{PaCO}_{2}$ partial pressure of carbon dioxide, AST aspartate-aminotransferase, ALT alanine-aminotransferase, LDH lactate dehydrogenase, $C R P$ $\mathrm{C}$-reactive protein

In case of missing data, sample sizes are given in brackets 
Table 2 Changes of clinical parameters from baseline to 6 months

\begin{tabular}{|c|c|c|c|c|c|c|c|}
\hline \multirow[t]{4}{*}{ Parameter [unit] } & \multirow{2}{*}{\multicolumn{3}{|c|}{$\begin{array}{l}\text { Placebo } \\
\text { Changes }(n=15)\end{array}$}} & \multicolumn{3}{|l|}{ Ambrisentan } & \multirow{4}{*}{$\begin{array}{l}95 \% \mathrm{Cl} \text { of } \\
\text { difference }\end{array}$} \\
\hline & & & & \multirow{2}{*}{\multicolumn{3}{|c|}{$\frac{\text { Changes }(n=17)}{\text { Baseline- } 6 \text { months }}$}} & \\
\hline & \multicolumn{3}{|c|}{ Baseline- 6 months } & & & & \\
\hline & Mean \pm SD & $95 \% \mathrm{Cl}$ & $n$ & Mean \pm SD & $95 \% \mathrm{Cl}$ & $n$ & \\
\hline \multicolumn{8}{|l|}{ Hemodynamics at rest } \\
\hline CVP $[\mathrm{mmHg}]$ & $-0.20 \pm 2.76$ & $(-1.73$ to 2.94$)$ & & $0.82 \pm 4.11$ & $(-1.29$ to 2.94$)$ & & $(-1.54$ to 3.59$)$ \\
\hline $\mathrm{mPAP}[\mathrm{mmHg}]$ & $-0.73 \pm 3.59$ & $(-2.72$ to 1.26$)$ & & $-1.00 \pm 6.40$ & $(-4.29$ to 2.29$)$ & & $(-4.09$ to 3.56$)$ \\
\hline PAWP $[\mathrm{mmHg}]$ & $0.13 \pm 3.20$ & $(-1.64$ to 1.91$)$ & & $1.24 \pm 5.31$ & $(-1.50$ to 3.97$)$ & & $(-2.12$ to 4.32$)$ \\
\hline $\mathrm{CO}[\mathrm{l} / \mathrm{min}]$ & $-0.26 \pm 1.11$ & $(-0.88$ to 0.36$)$ & & $0.58 \pm 1.17$ & $(-0.03$ to 1.18$)$ & & (0.01 to 1.66$)$ \\
\hline $\mathrm{Cl}\left[\mathrm{l} / \mathrm{min} / \mathrm{m}^{2}\right]$ & $-0.31 \pm 0.71$ & $(-0.71$ to 0.08$)$ & & $0.36 \pm 0.66$ & (0.01 to 0.71$)$ & (16) & (0.18 to 1.18$)$ \\
\hline $\mathrm{SvO}_{2}[\%]$ & $-3.48 \pm 12.26$ & ( -10.89 to 3.93$)$ & (13)\# & $-2.79 \pm 7.56$ & $(-7.60$ to 2.01$)$ & (12)\# & $(-7.83$ to 9.20$)$ \\
\hline PVR [WU] & $0.02 \pm 0.76$ & $(-0.48$ to 0.43$)$ & & $-0.59 \pm 0.79$ & $(-1.05$ to -0.14$)$ & & $(-1.25$ to -0.17$)$ \\
\hline \multicolumn{8}{|l|}{ Hemodynamics at peak exercise } \\
\hline $\mathrm{mPAP}[\mathrm{mmHg}]$ & $1.08 \pm 7.39$ & $(-3.39$ to 5.54$)$ & (13) & $-0.73 \pm 6.23$ & $(-4.18$ to 2.71$)$ & (15) & $(-7.10$ to 3.48$)$ \\
\hline PAWP $[\mathrm{mmHg}]$ & $0.85 \pm 6.3$ & $(-2.96$ to 4.65$)$ & (13) & $4.93 \pm 6.52$ & (1.16 to 8.69$)$ & (14) & $(-1.01$ to 9.17$)$ \\
\hline $\mathrm{CO}[\mathrm{l} / \mathrm{min}]$ & $-0.05 \pm 1.46$ & ( -0.94 to 0.83$)$ & (13) & $1.24 \pm 1.47$ & (0.43 to 2.05$)$ & (15) & (0.15 to 2.44$)$ \\
\hline $\mathrm{Cl}\left[\mathrm{l} / \mathrm{min} / \mathrm{m}^{2}\right]$ & $-0.45 \pm 1.36$ & $(-1.27$ to 0.37$)$ & (13) & $0.70 \pm 0.81$ & (0.25 to 1.15$)$ & (15) & (0.29 to 2.00$)$ \\
\hline $\mathrm{SvO}_{2}[\%]$ & $-28.35 \pm 10.5$ & $(-35.41$ to -21.30$)$ & (11) & $-30.05 \pm 17.73$ & $(-42.73$ to -17.37$)$ & (10) & $(-14.85$ to 11.46$)$ \\
\hline Workload [Watt] & $1.92 \pm 16.01$ & $(-7.75$ to 11.60$)$ & (13) & $5.00 \pm 16.9$ & $(-4.36$ to 14.36$)$ & (15) & $(-9.77$ to 15.93$)$ \\
\hline HR max [b/min] & $6.00 \pm 18.74$ & $(-5.32$ to 17.32$)$ & (13) & $7.47 \pm 12.01$ & (0.82 to 14.12 ) & (15) & ( -10.59 to 13.53$)$ \\
\hline PVR [WU] & $-0.003 \pm 0.34$ & $(-0.21$ to 0.21$)$ & (13) & $-0.84 \pm 0.48$ & $(-1.12$ to -0.57$)$ & (14) & $(-1.17$ to -0.51$)$ \\
\hline $\operatorname{TPR}\left[\mathrm{mmHg} \min \mathrm{L}^{-1}\right]$ & $0.16 \pm 0.63$ & $(-0.22$ to 0.54$)$ & (13) & $-0.56 \pm 0.48$ & $(-0.83$ to -0.29$)$ & (15) & $(-1.16$ to -0.29$)$ \\
\hline \multicolumn{8}{|l|}{ 6MWD } \\
\hline $6 \mathrm{MWD}[\mathrm{m}]$ & $-16.53 \pm 77.32$ & $(-59.35$ to 26.29$)$ & & $21.53 \pm 34.6$ & (3.74 to 39.31 ) & & ( -4.30 to 80.43$)$ \\
\hline Borg dyspnea score & $0.08 \pm 1.54$ & $(-0.77$ to 0.93$)$ & & $0.62 \pm 1.7$ & $(-0.25$ to 1.50$)$ & & ( -0.63 to 1.72$)$ \\
\hline $\mathrm{SaO}_{2}$ after $6 \mathrm{MWD}[\%]$ & $1.30 \pm 5.1$ & $(-2.35$ to 4.95$)$ & (10)\# & $2.07 \pm 5.7$ & $(-1.22$ to 5.37$)$ & (14) & $(-3.92$ to 5.46$)$ \\
\hline HR after 6MWD [/min] & $6.83 \pm 24.97$ & $(-9.04$ to 22.70$)$ & (12)\# & $-1.53 \pm 18.43$ & $(-11.74$ to 8.68$)$ & (15) & $(-25.56$ to 8.83$)$ \\
\hline \multicolumn{8}{|l|}{ Quality of life SF-36 } \\
\hline Physical functioning & $-2.00 \pm 25.20$ & $(-15.96$ to 11.96$)$ & & $-7.65 \pm 21.66$ & $(-18.78$ to 3.49$)$ & & $(-22.56$ to 11.27$)$ \\
\hline Physical role functioning & $13.33 \pm 38.81$ & (- 8.12 to 34.82$)$ & & $-10.29 \pm 42.44$ & $(-32.12-11.53)$ & & $(-53.14$ to 5.88$)$ \\
\hline Bodily pain & $-2.47 \pm 28.19$ & $(-18.08$ to 13.15$)$ & & $-9.29 \pm 23.87$ & $(-21.57$ to 2.98$)$ & & $(-25.62$ to 11.97$)$ \\
\hline General health perceptions & $0.20 \pm 19.39$ & $(-10.54$ to 10.94$)$ & & $-2.71 \pm 10.62$ & $(-8.17$ to 2.75$)$ & & $(-14.01$ to 8.20$)$ \\
\hline Vitality & $-5.00 \pm 16.37$ & $(-14.06$ to 4.06$)$ & & $-3.53 \pm 12.34$ & (- 9.88 to 2.82$)$ & & (- 8.92 to 11.86$)$ \\
\hline Social role functioning & $0.87 \pm 27.69$ & $(-14.47$ to 16.20$)$ & & $-2.18 \pm 19.21$ & $(-12.05$ to 7.70$)$ & & $(-20.08$ to 13.99$)$ \\
\hline Emotional role functioning & $8.80 \pm 49.68$ & $(-18.71$ to 36.31$)$ & & $-9.82 \pm 36.76$ & ( -28.73 to 9.08$)$ & & $(-49.93$ to 12.68$)$ \\
\hline Mental health & $-3.73 \pm 11.85$ & $(-10.30$ to 2.83$)$ & & $-4.47 \pm 10.94$ & $(-10.10$ to 1.16$)$ & & ( -8.97 to 7.49$)$ \\
\hline Physical health score & $0.87 \pm 16.01$ & $(-8.00$ to 9.73$)$ & & $-6.71 \pm 12.17$ & $(-12.97$ to -0.45$)$ & & $(-17.77$ to 2.62$)$ \\
\hline Mental health score & $0.27 \pm 19.77$ & $(-10.68$ to 11.22$)$ & & $-4.65 \pm 9.47$ & $(-9.51$ to 0.22$)$ & & $(-15.89$ to 6.06$)$ \\
\hline mRSS & $0.73 \pm 2.15$ & $(-0.46$ to 1.93$)$ & & $0.24 \pm 0.97$ & $(-0.26$ to 0.73$)$ & & $(-1.68$ to 0.68$)$ \\
\hline \multicolumn{8}{|l|}{ Lung function } \\
\hline FVC [\%] & $-1.04 \pm 5.60$ & $(-4.15$ to -2.06$)$ & & $-3.31 \pm 5.56$ & $(-6.27$ to -0.34$)$ & (16) & $(-6.37$ to 1.84$)$ \\
\hline FEV1 [L] & $-0.06 \pm 0.20$ & $(-0.17$ to 0.05$)$ & & $-0.11 \pm 0.21$ & $(-0.22$ to 0.01$)$ & (16) & $(-0.20$ to 0.11$)$ \\
\hline FEV1\% VC max [\%] & $-0.67 \pm 4.50$ & $(-3.16$ to 1.82$)$ & & $-4.17 \pm 7.12$ & $(-7.97$ to -0.38$)$ & (16) & (- 7.91 to 0.91$)$ \\
\hline PEF [l/s] & $-0.22 \pm 1.76$ & $(-1.12$ to 0.76$)$ & & $0.01 \pm 1.15$ & $(-0.61$ to 0.62$)$ & (16) & $(-0.86$ to 1.31$)$ \\
\hline TLC [l] & $-0.03 \pm 0.36$ & $(-0.23$ to 0.17$)$ & & $-0.06 \pm 0.37$ & $(-0.26$ to 0.14$)$ & (16) & $(-0.30$ to 0.24$)$ \\
\hline
\end{tabular}


Table 2 Changes of clinical parameters from baseline to 6 months (Continued)

\begin{tabular}{|c|c|c|c|c|c|c|c|}
\hline \multirow[t]{4}{*}{ Parameter [unit] } & \multicolumn{3}{|l|}{ Placebo } & \multicolumn{3}{|l|}{ Ambrisentan } & \multirow{4}{*}{$\begin{array}{l}95 \% \mathrm{Cl} \text { of } \\
\text { difference }\end{array}$} \\
\hline & \multicolumn{3}{|c|}{ Changes $(n=15)$} & \multicolumn{3}{|l|}{ Changes $(n=17)$} & \\
\hline & \multicolumn{3}{|c|}{ Baseline- 6 months } & \multicolumn{3}{|c|}{ Baseline- 6 months } & \\
\hline & Mean \pm SD & $95 \% \mathrm{Cl}$ & $n$ & Mean \pm SD & $95 \% \mathrm{Cl}$ & $n$ & \\
\hline Residual volume [l] & $0.05 \pm 0.37$ & $(-0.16$ to 0.25$)$ & & $-0.03 \pm 0.33$ & $(-0.20$ to 0.15$)$ & (16) & $(-0.33$ to 1.83$)$ \\
\hline $\mathrm{DLCO}[\mathrm{mmol} / \mathrm{min} / \mathrm{kPa}]$ & $-0.45 \pm 1.70$ & $(-1.48$ to 0.57$)$ & (13) & $-0.32 \pm 1.44$ & $(-1.06$ to 0.42$)$ & & $(-1.04$ to 1.30$)$ \\
\hline DLCO \% predicted & $-0.44 \pm 1.84$ & $(-1.55$ to 0.67$)$ & (13) & $1.19 \pm 1.81$ & (0.26 to 2.12$)$ & (17) & (25.10 to 30.04$)$ \\
\hline $\mathrm{SaO}_{2}[\%]$ & $0.15 \pm 1.86$ & $(-0.88$ to 1.18$)$ & & $-0.62 \pm 1.50$ & $(-1.39$ to 0.16$)$ & & $(-1.99$ to 0.44$)$ \\
\hline $\mathrm{PaO}_{2}[\mathrm{mmHg}]$ & $1.69 \pm 9.95$ & $(-3.82$ to 7.21$)$ & & $-4.88 \pm 7.60$ & $(-8.78$ to -0.97$)$ & & $(-12.92$ to -0.22$)$ \\
\hline $\mathrm{PaCO}_{2}[\mathrm{mmHg}]$ & $-0.03 \pm 2.63$ & $(-1.48$ to 1.43$)$ & & $-0.65 \pm 2.77$ & $(-2.08$ to 0.77$)$ & & $(-2.58$ to 1.33$)$ \\
\hline \multicolumn{8}{|l|}{ Echocardiography } \\
\hline Estimated sPAP [mmHg] & $-0.93 \pm 6.08$ & $(-4.30$ to 2.43$)$ & & $-0.82 \pm 4.46$ & $(-3.11$ to 1.47$)$ & & $(-3.71$ to 3.93$)$ \\
\hline RA area $\left[\mathrm{cm}^{2}\right]$ & $-0.47 \pm 4.07$ & $(-2.72$ to 1.79$)$ & & $1.65 \pm 2.67$ & $(0.28$ to 3.01$)$ & & $(-0.34$ to 4.57$)$ \\
\hline $\mathrm{RV}$ area $\left[\mathrm{cm}^{2}\right]$ & $-0.80 \pm 3.05$ & $(-2.49$ to 0.89$)$ & & $-0.15 \pm 3.46$ & $(-1.93$ to 1.63$)$ & & $(-1.72$ to 3.02$)$ \\
\hline TAPSE $[\mathrm{cm}]$ & $-0.19 \pm 0.54$ & $(-0.49$ to 0.11$)$ & & $0.12 \pm 0.41$ & $(-0.09$ to 0.33$)$ & & $(-0.04$ to 0.65$)$ \\
\hline \multicolumn{8}{|l|}{ Laboratory } \\
\hline Hemoglobin $[\mathrm{g} / \mathrm{dl}]$ & $0.19 \pm 0.68$ & $(-0.19$ to 0.56$)$ & & $-0.59 \pm 0.86$ & $(-1.03$ to -0.15$)$ & & $(-1.34$ to -0.21$)$ \\
\hline Hematocrit $[1 / 1]$ & $0.00 \pm 0.02$ & $(-0.009$ to 0.02$)$ & & $-0.01 \pm 0.02$ & $(-0.03$ to -0.0001$)$ & & $(-0.03$ to 0.00$)$ \\
\hline Platelets [100/nl] & $-0.08 \pm 0.39$ & $(-0.29$ to 0.14$)$ & & $-0.15 \pm 0.37$ & $(-0.34$ to 0.04$)$ & & $(-0.35$ to 0.20$)$ \\
\hline Creatinine [mg/dl] & $-0.03 \pm 0.09$ & $(-0.08$ to 0.02$)$ & & $-0.04 \pm 0.11$ & $(-0.09$ to 0.02$)$ & & $(-0.08$ to 0.07$)$ \\
\hline Potassium [mmol/l] & $0.09 \pm 0.40$ & $(-0.13$ to 0.31$)$ & & $-0.06 \pm 0.62$ & $(-0.38$ to 0.26$)$ & & $(-0.53$ to 0.24$)$ \\
\hline AST [U/I] & $-4.40 \pm 13.94$ & $(-12.12$ to 3.32$)$ & & $3.59 \pm 7.96$ & $(-0.51$ to 7.68$)$ & & $(-0.08$ to 16.06$)$ \\
\hline $\mathrm{ALT}[\mathrm{U} / \mathrm{I}]$ & $-4.93 \pm 15.01$ & $(-13.25$ to 3.38$)$ & & $5.12 \pm 7.83$ & (1.09 to 9.15$)$ & & (1.56 to 18.55$)$ \\
\hline $\mathrm{LDH}[\mathrm{U} / \mathrm{I}]$ & $-7.00 \pm 27.36$ & $(-22.15$ to 8.15$)$ & & $2.82 \pm 29.57$ & $(-12.38$ to 18.03$)$ & & $(-10.84$ to 30.49$)$ \\
\hline CK $[\mathrm{U} / \mathrm{l}]$ & $9.21 \pm 40.50$ & $(-14.17$ to 32.60$)$ & (14) & $5.53 \pm 36.80$ & $(-13.39$ to 24.45$)$ & & $(-32.10$ to 24.73$)$ \\
\hline CRP $[\mathrm{mg} / \mathrm{ll}]$ & $-1.08 \pm 3.52$ & $(-3.03$ to 0.87$)$ & & $-2.71 \pm 12.17$ & $(-8.97$ to 3.54$)$ & & $(-8.29$ to 5.03$)$ \\
\hline NTproBNP [pg/ml] & $31.00 \pm 85.83$ & $(-20.87$ to 82.87$)$ & (13) & $-15.63 \pm 207.48$ & $(-126.18$ to 94.93$)$ & (16) & $(-172.95$ to 79.70$)$ \\
\hline
\end{tabular}

SD standard deviation, CVP central venous pressure, mPAP mean pulmonary arterial pressure, $P A W P$ pulmonary capillary wedge pressure, $C O$ cardiac output, $C I$ cardiac index, SVO2 venous oxygen saturation, PVR pulmonary vascular resistance, WU Wood Units, HR heart rate, $b$ beats, min minute, TPR total pulmonary resistance, 6MWD Six-minute walking distance, SaO2 oxygen saturation, HR heart rate, mRSS modified Rodnan Skin Score, FVC forced vital capacity, FEV1 forced expiratory volume in first second, VC vital capacity, PEF peak expiratory flow, TLC total lung capacity, DLCO diffusing capacity of the lung for carbon monoxide, $\mathrm{SaO} 2$ oxygen saturation, $\mathrm{PaO} 2$ partial pressure of oxygen, $\mathrm{PaCO} 2$ partial pressure of carbon dioxide, SPAP systolic pulmonary arterial pressure, RA right atrial, $R \mathrm{~V}$ right ventricular, TAPSE tricuspid annular plane systolic excursion, $A S T$ aspartate-aminotransferase, $A L T$ alanine-aminotransferase, $L D H$ lactate dehydrogenase, $C K$ creatine kinase, CRP C-reactive protein, NTproBNP N-terminal pro-brain natriuretic peptide In case of missing data, sample sizes are given in brackets \#Values with more than $20 \%$ missing data

Both $\mathrm{PH}$ patients in the ambrisentan group were categorized as newly developed manifest $\mathrm{PH}$ due to left heart diseases characterized by an increased resting PAWP of $>15 \mathrm{mmHg}$ (Fig. 2). One patient was a 71-year-old female with systemic arterial hypertension. Her resting mPAP at baseline increased from 24 to $29 \mathrm{mmHg}$ and resting PAWP from 11 to $18 \mathrm{mmHg}$ after 6 months. Subsequent examinations including left heart catheterization showed a progression of left ventricular diastolic dysfunction. The second patient with manifest $\mathrm{PH}$ after 6 months was a 79-year-old female with a resting $\mathrm{mPAP}$ of $18 \mathrm{mmHg}$ at baseline and a resting PAWP of $5 \mathrm{mmHg}$, which markedly increased to an mPAP of $36 \mathrm{mmHg}$ after 6 months with a resting PAWP of $22 \mathrm{mmHg}$. Left heart catheterization revealed a coronary artery disease.

The secondary endpoints $\mathrm{CO}$ and $\mathrm{CI}$ significantly increased in the ambrisentan group compared to those in the placebo group which tended to decrease, both at rest ( $p=0.047, p=0.010$, respectively) and at peak exercise $(p=0.028, p=0.015$, respectively, Fig. 3). Furthermore, change of TPR significantly differed between groups, with a decrease of TPR in the ambrisentan group and an increase in the placebo group $(p=0.022$, Table 2$)$. There were no significant changes of PAWP in the ambrisentan group compared to the placebo group at rest $(p=0.478)$ or at maximal exercise $(p=0.111)$. Compared to the placebo 

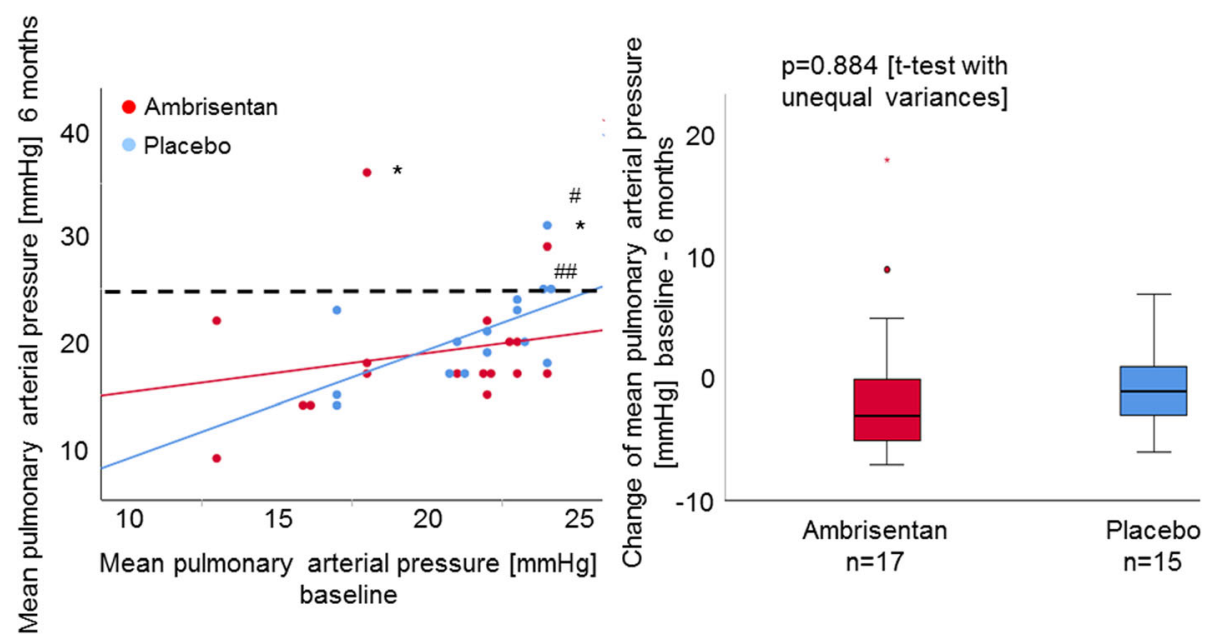

Fig. 2 Changes of mPAP over 6 months. No patients at baseline had a resting mPAP of $\geq 25 \mathrm{mmHg}$. After 6 months, 2 patients in the ambrisentan group developed a resting mPAP of $>25 \mathrm{mmHg}$. The dotted line indicates a resting mPAP of $25 \mathrm{mmHg}$. *: Two patients in the ambrisentan group had a resting PAWP of $>15 \mathrm{mmHg}$ after 6 months; they were reclassified as PH due to left heart disease. \#: Three patients in the placebo group developed a resting mPAP of $\geq 25 \mathrm{mmHg}$ at month 6 with a resting PAWP of $\leq 15 \mathrm{mmHg}$; thus, they were diagnosed as having SSc-APAH after 6 months. The mean change of resting mPAP over 6 months in the ambrisentan group was $-1 \pm 6.4 \mathrm{mmHg}$, and that in the placebo group was $-0.73 \pm 3.59 \mathrm{mmHg}$. The changes between the two groups were not significantly different $(p=0.884)$. Ambrisentan did not significantly decrease the MPAP at rest over 6 months compared to placebo

group, PVR in the ambrisentan group significantly decreased over 6 months both at rest (Fig. 4) and at maximal exercise $(p=0.012, p<0.0001$, respectively). There was no significant difference in MPAP $(p=0.494)$, workload, and heart rate at peak exercise between the two groups.

WHO functional class, 6-min walking test, quality of life, and skin fibrosis (Table 2)

At 6 months, 17 patients $(100 \%)$ in the ambrisentan group and 13 patients $(86.7 \%)$ in the placebo group were in WHO-FC II. The remaining 2 patients in the placebo group were in WHO-FC III.
After 6 months, the mean 6MWD improved by 21.53 $\mathrm{m}$ in the ambrisentan group and decreased by $16.53 \mathrm{~m}$ in the placebo group ( $p=0.095$; Table 2$)$. No differences were detected regarding quality of life and skin fibrosis. Sensitivity analysis of patients with dcSSc did not reveal different results.

\section{Lung function, transthoracic echocardiography, and laboratory data (Table 2)}

The changes in predicted DLCO showed a decrease in the placebo group and an increase in the ambrisentan
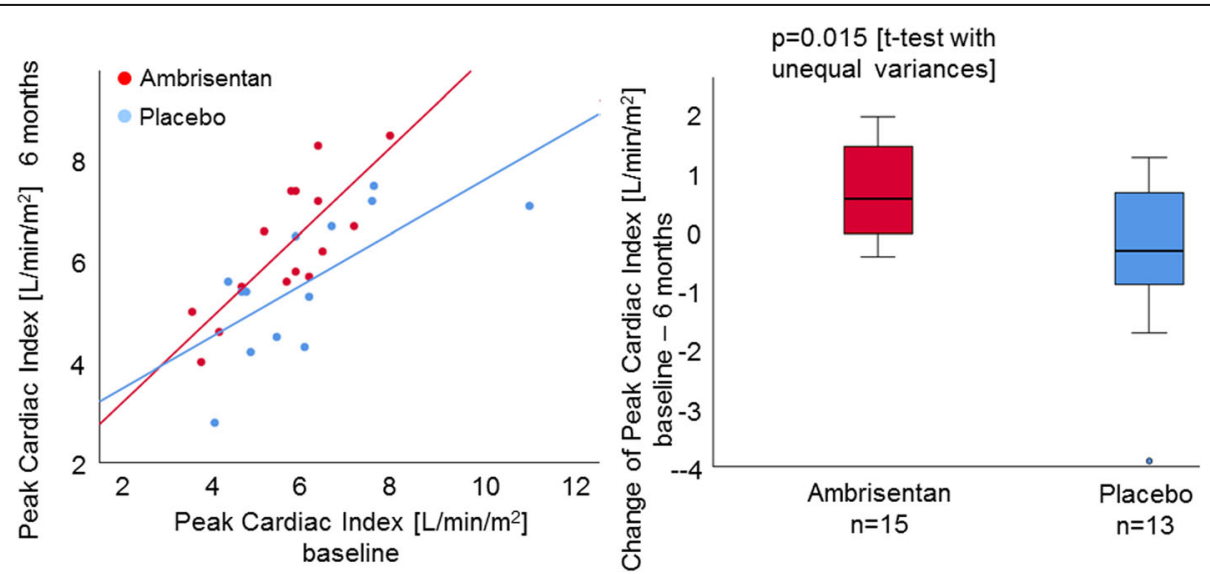

Fig. 3 Changes of peak Cl over 6 months. The mean change of $\mathrm{Cl}$ at maximal exercise over 6 months in the ambrisentan group was $0.70 \pm 0.81 \mathrm{~L} /$ $\mathrm{min} / \mathrm{m}^{2}$ and that in the placebo group was $-0.45 \pm 1.36 \mathrm{~L} / \mathrm{min} / \mathrm{m}^{2}$. Ambrisentan significantly increased the $\mathrm{Cl}$ at maximal exercise over $6 \mathrm{months}$ compared to placebo $(p=0.015)$ 


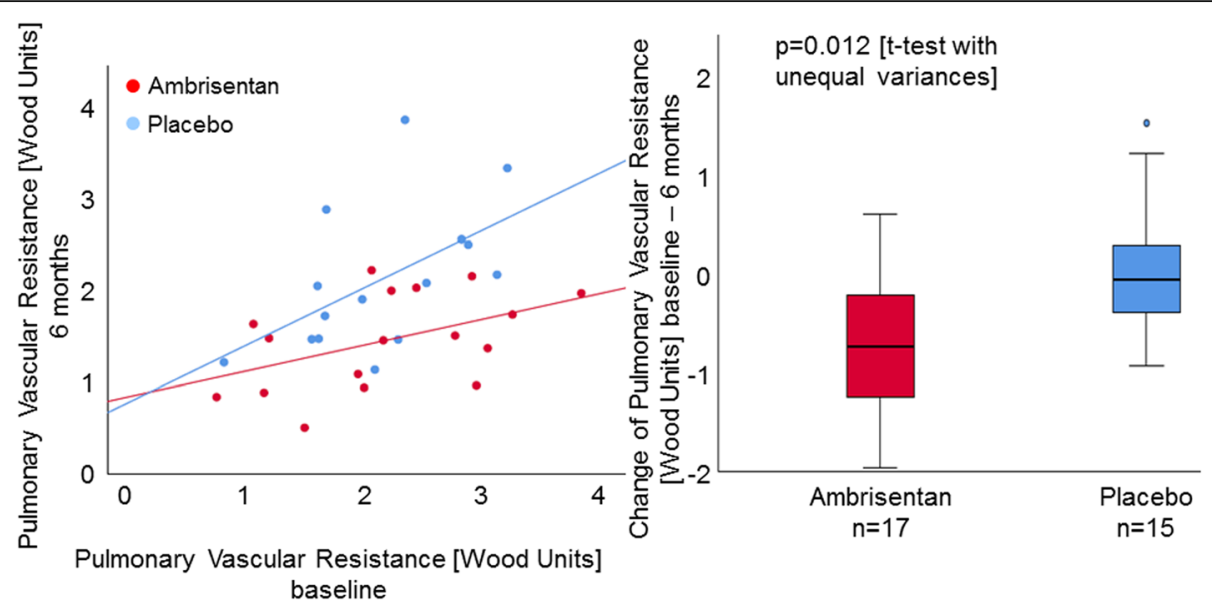

Fig. 4 Changes of PVR at rest over 6 months. Ambrisentan patients had on average a lower PVR at 6 months compared to placebo. The mean change of PVR at rest over 6 months in the ambrisentan group was $-0.70 \pm 0.78 \mathrm{WU}$ and that in the placebo group was $0.01 \pm 0.71 \mathrm{WU}$. Ambrisentan significantly decreased the PVR at rest over 6 months compared to placebo $(p=0.012)$

group (95\% confidence interval of the difference $0.25-$ $3.0 \%$, Table 2). No changes between the two groups were recorded with regard to other parameters of pulmonary function. A change in the partial pressure of oxygen $\left(\mathrm{PaO}_{2}\right)$ was recorded, with a decrease in the ambrisentan group, whereas it slightly increased in the placebo group.

Table 3 Adverse events

\begin{tabular}{lll}
\hline Event & $\begin{array}{l}\text { Placebo } \\
(N=19)\end{array}$ & $\begin{array}{c}\text { Ambrisentan } \\
(N=19)\end{array}$ \\
& Number of patients (percent) \\
\hline Patient with at least 1 adverse event & $17(89.5)$ & $17(89.5)$ \\
Headache & $6(31.58)$ & $6(31.58)$ \\
Edema & $4(21.05)$ & $8(42.11)$ \\
Dizziness & $6(31.58)$ & $0(0)+$ \\
Diarrhea & $2(10.53)$ & $4(21.05)$ \\
Nausea & $3(15.79)$ & $2(10.53)$ \\
Paresthesia & $0(0)$ & $4(21.05)$ \\
Coronary artery disease & $3(15.79)$ & $1(5.26)$ \\
Hypotension & $2(10.53)$ & $2(10.53)$ \\
Epistaxis & $1(5.26)$ & $3(15.79)$ \\
Serious adverse events & & \\
Lower jaw fracture & & 0 \\
Angina Pectoris & 0 & 0 \\
Coronary artery disease & 1 & 0 \\
Gastrointestinal infection & 1 & 0 \\
Lymphangitis & 1 & 1 \\
Raynaud & 1 & 0
\end{tabular}

The adverse events listed here are those that occurred in at least $10 \%$ of patients (total) during the course of the study †Statistically significant at level 0.05

*All serious adverse events fulfilled the criterion of hospitalization
Parameters of right heart size and function did not show differences between groups.

Patients receiving ambrisentan had a not clinically relevant drop in hemoglobin concentration $(-0.59 \pm$ $0.86 \mathrm{~g} / \mathrm{dl}, 95 \%$ confidence interval -1.03 to -0.15 ) compared to placebo. No differences were identified regarding other laboratory parameters, including those concerning renal failure, liver damage, and NTproBNP levels.

\section{Safety and tolerability (Table 3 )}

Most of the adverse events were of mild or moderate intensity in both groups and are summarized in Table 3. Serious adverse events were more frequently reported for the placebo group (Table 3), with all events requiring hospitalization, but being resolved at the end of the study.

\section{Discussion}

To the best of our knowledge, EDITA is the first randomized, controlled study to assess the safety and efficacy of an early treatment with a PAH-targeted drug in patients with SSc and mildly elevated mPAP (mPAP 21$24 \mathrm{mmHg}$ ) and/or exercise $\mathrm{PH}$ in comparison with placebo. Patients did not significantly differ in the primary endpoint change of mPAP during the study. However, the secondary hemodynamic endpoints as peak TPR as well as right heart function (CO, CI), and PVR significantly improved in the ambrisentan group, both at rest and at peak exercise compared to placebo. No patients in the ambrisentan group but three patients from the placebo group progressed to SSc-associated PAH within 6 months of treatment (although for two patients the progression was only minimal). Ambrisentan was well tolerated, with a favorable safety profile. 


\section{Effects of ambrisentan in patients with SSc and early pulmonary vasculopathy}

While the primary endpoint of the study was not met, parameters of right ventricular (RV) function and PVR at rest and during exercise showed significant improvements during the study. Data from large registries have already shown the prognostic importance of $\mathrm{CO}, \mathrm{CI}$, and PVR at rest $[25,26]$. These three parameters showed a significant improvement in the ambrisentan group after 6 months in our study, compared to placebo.

Besides resting values, exercise hemodynamics are able to unmask early RV dysfunction and vascular remodeling, especially in patients with SSc and mildly elevated mPAP, who usually display normal right heart function at rest $[9,27]$. In our study, improvements of $\mathrm{CO}$ and $\mathrm{CI}$ at peak exercise were even more pronounced than changes observed at rest. The increase of $\mathrm{CO}$ and $\mathrm{CI}$ during exercise (also called RV output reserve) is able to provide useful information regarding prognosis of patients with pulmonary vascular diseases $[28,29]$.

The finding that change of TPR slope significantly differed between groups and that only patients in the placebo group presented with a manifest SSc-APAH during follow-up, whereas two patients in the ambrisentan group developed left heart disease $\mathrm{PH}$, may be a hint for the beneficial effect of ambrisentan on the pulmonary vasculature, though the primary endpoint of mPAP change was not met. As two patients in the intervention group presented with left heart disease $\mathrm{PH}$ at the end of the study, though they did not present with significant left heart disease at baseline, treatment with ambrisentan may potentially lead to the unmasking and progression of left heart disease in patients with SSc [30]. Furthermore, pulmonary fibrosis has to be taken into account when initiating treatment with ambrisentan, as ambrisentan may lead to worsening of $\mathrm{PaO}_{2}$ [30], as found in our cohort, though not clinically relevant. Patients who are eligible for an early treatment with PAH-targeted drugs have therefore to be carefully selected. Furthermore, hemoglobin as well as hepatic values have to be thoroughly monitored. Further, larger-scaled studies are needed to investigate the effect of early PAH-targeted treatment in SSc patients with mildly elevated mPAP and/or exercise $\mathrm{PH}$ and its impact on the pulmonary vascular system and right heart function.

\section{Comparison with previous reports on treatment with PAH-targeted drugs in patients with SSC and early pulmonary vasculopathy}

Two uncontrolled small open-label reports were previously performed, one with ambrisentan [15] and one with bosentan [16]. Our data are in line with those published by Saggar et al. on 12 patients with exercise induced $\mathrm{PH}<25 \mathrm{mmHg}$ at rest and $>30 \mathrm{mmHg}$ at peak exercise receiving ambrisentan treatment for 24 weeks. After 24 weeks of treatment, the authors did not find a significant decrease of resting $\mathrm{MPAP}(p=0.65)$ but a remarkable improvement of $\mathrm{CO}$ at rest and during exercise ( $p=0.01$ and $p=0.006$ respectively) and of PVR during exercise $(p=0.003)$.

Kovacs et al. assessed in a retrospective uncontrolled study 10 patients with mildly elevated mPAP (21-24 $\mathrm{mmHg}$ ) receiving bosentan for 24 weeks. Apart from an improvement in right heart function ( $\mathrm{CO}$ at rest $p=0.05)$, they reported a significant reduction of mPAP at rest and peak exercise ( $p=0.03$ and $p=0.02$, respectively) after 24 weeks of bosentan treatment [16]. Congruently with the results of Kovacs and Saggar, we did not find significant improvements in 6MWD, QoL, and WHO-FC. Since more than $70 \%$ of our cohort had 6MWD $>400 \mathrm{~m}$ and WHO-FC II at baseline, our findings are concordant with the results of a post hoc analysis of the ambrisentan in $\mathrm{PAH}$, randomized, double-blind, placebo-controlled, multicenter efficacy study 1 and 2 (ARIES)-1 and ARIES-2 studies, which reported that ambrisentan had a greater effect on 6MWD and WHO-FC in patients with more severe PAH [31].

\section{Clinical implication and safety}

Pulmonary vascular abnormalities in PVR have been shown to be already remarkable even in patients with only mild symptoms [32] and may precede an overt disease [33]. The improvement in PVR observed in our cohort might indicate that active treatment with ambrisentan is able to prevent the progressive vascular remodeling in SSc patients with an early form of pulmonary vasculopathy.

Ambrisentan treatment has shown a good safety profile, which is in line with the current analysis of safety and tolerability [34, 35]. Undesirable effects of ambrisen$\tan$ recorded in our study were a clinically not relevant drop of hemoglobin concentration and $\mathrm{PaO}_{2}$. The reduction of hemoglobin found in our study may be due to a class effect of all endothelin receptor antagonists, and the most probable cause might be fluid retention [36]. A non-dose-dependent change in hemoglobin values was also found in the first 12 weeks of the ARIES- 1 and ARIES-2 studies, with stabilization during the subsequent 24 weeks [11]. Long-term extension of the ARIES studies confirmed that hemoglobin levels tended to stabilize over time [37].

\section{Limitations}

Our study may have been biased due to the inclusion criteria of including patients with mildly elevated mPAP both at rest and during exercise. The distribution of these entities may have influenced the results, though it did not differ between the two groups. As there is 
growing evidence that both states with mildly elevated pressures at rest as well as during exercise bear the risk of developing manifest pulmonary vascular disease, we included both phenotypes into the study. Furthermore, a longer study period of more than 6 months may have helped to interpret the results, as longer time intervals could have led to more distinct changes in pulmonary hemodynamics. As two patients of the placebo group with manifest $\mathrm{PAH}$ at follow-up increased only minimal, the changes are too small to draw definite conclusions. Larger-scaled studies with longer observation periods are needed to further investigate this issue.

Among our study cohort, two patients developed left heart disease PH during the study period, which was based on PAWP at rest and during exercise. While it would be preferable to clearly distinguish the effects of targeted treatment on the pulmonary vasculature, it is hardly possible to exclude interfering diseases such as left heart and lung disease. Patients in our study were only enrolled in the absence of significant left heart or lung disease at baseline to limit determining factors of mPAP elevation and development of manifest $\mathrm{PH}$. To further characterize patients with $\mathrm{PH}$ due to left heart disease, the assessment of the diastolic pressure gradient and echocardiographic parameters of diastolic dysfunction would have been desirable. The higher frequency of patients with lcSSc and shorter duration of SSc in the ambrisentan group may have influenced the results, though sensitivity analysis of patients with dcSSc did not show different results. "Development of PAH" as a primary endpoint would have required a large sample size, which would not have been feasible in a single-center study.

\section{Conclusions}

Although the primary endpoint was not met (change in mPAP over 6 months), ambrisentan was associated with significant improvements of secondary endpoints, such as peak TPR, PVR, CO, and CI both at rest and at peak exercise. Ambrisentan showed good safety and tolerability in this patient cohort. The potential risks of ambrisentan of unmasking left heart disease, drop of hemoglobin, and $\mathrm{PaO}_{2}$ have to be taken into account before treatment initiation and thoroughly monitored during treatment. All SSc patients included in this study had symptoms such as shortness of breath with a WHO-FC II-III, some of them revealed already an impaired RV function at rest and during exercise. Thus, these patients had already a cardiopulmonary disease and further treatment studies are mandatory. Determinants of treatment response as well as development of comorbidities should also be a focus of future studies. The results of this study will be helpful to design future trials.

\section{Abbreviations}

6MWD: 6-min walking distance; Cl: Cardiac index; CO: Cardiac output; dcSSc: Diffuse cutaneous systemic sclerosis; DLCO: Diffusion capacity of the lung; ECG: Electrocardiogram; FVC: Forced vital capacity; HRCT: Highresolution computed tomography; ICSSc: Limited cutaneous systemic sclerosis; mPAP: Mean pulmonary arterial pressure; mRSS: Modified Rodnan skin score; NTproBNP: N-terminal fraction of the pro-brain natriuretic peptide; PAH: Pulmonary arterial hypertension; PAWP: Pulmonary arterial wedge pressure; PH: Pulmonary hypertension; PVR: Pulmonary vascular resistance; QoL: Quality of life; RHC: Right heart catheterization; RV: Right ventricular; SSc: Systemic sclerosis; $\mathrm{SVO}_{2}$ : Venous oxygen saturation; TPG: Transpulmonary gradient; TPR: Total pulmonary resistance; TnT: Troponin T; WHO-FC: World Health Organization functional class

\section{Acknowledgements}

Not applicable.

\section{Authors' contributions}

ZP made substantial contributions to study conception and design and to analysis and interpretation of data. AMM made substantial contributions to analysis and interpretation of data. NBe made substantial contributions to study conception and design and to analysis and interpretation of data. CAE made substantial contributions to study conception and design and to analysis and interpretation of data. NoB made substantial contributions to acquisition, analysis, and interpretation of data. EB made substantial contributions to analysis and interpretation of data. AC made substantial contributions to analysis and interpretation of data. GC made substantial contributions to analysis and interpretation of data. CPD made substantial contributions to analysis and interpretation of data. OD made substantial contributions to analysis and interpretation of data. BE made substantial contributions to acquisition, analysis, and interpretation of data. CF made substantial contributions to analysis and interpretation of data. SH made substantial contributions to acquisition, analysis, and interpretation of data. PX made substantial contributions to acquisition, analysis, and interpretation of data. HML made substantial contributions to acquisition, analysis, and interpretation of data. EG made substantial contributions to study conception and design and data acquisition and to analysis and interpretation of data. All authors were involved in drafting the article or revising it critically for important intellectual content and gave final approval of the version of the article to be published.

\section{Funding}

The study was funded by a grant from GlaxoSmithKline. The company had no influence on the study design, data analysis, and interpretation.

\section{Availability of data and materials}

The data is available from the corresponding author upon reasonable request.

\section{Ethics approval and consent to participate}

A written informed consent was obtained from all patients in accordance with the Declaration of Helsinki. The approval of the ethics committee of the Medical Faculty Heidelberg was obtained (AFmo-407/2014).

\section{Consent for publication}

Not applicable.

\section{Competing interests}

$\mathrm{ZP}, \mathrm{AMM}, \mathrm{CAE}, \mathrm{EB}, \mathrm{AC}, \mathrm{CF}$, and $\mathrm{PX}$ declare that they have no competing interests. NBe has received speaker honoraria from Actelion pharmaceuticals, not related to this study. NoB has received speaker honoraria from Actelion pharmaceuticals, not related to this study. GC reports research support, consultancy fees, and speaker honoraria from Actelion pharmaceuticals. CPD reports speaker honoraria from Actelion pharmaceuticals. OD reports research support and consultancy fees from Actelion pharmaceuticals. BE reports speaker honoraria from Actelion pharmaceuticals. SH reports speaker honoraria from Actelion pharmaceuticals. HML reports speaker and consultancy fees from Actelion pharmaceuticals. EG has received speaker honoraria and advisory board fees from Actelion pharmaceuticals. 


\section{Author details}

${ }^{1}$ Centre for Pulmonary Hypertension, Thoraxklinik at Heidelberg University Hospital, Röntgenstraße 1, 69126 Heidelberg, Germany. ${ }^{2}$ Translational Lung Research Center Heidelberg (TLRC), Member of the German Center for Lung Research (DZL), Heidelberg, Germany. ${ }^{3}$ IRCCS SDN Research Institute, Naples, Italy. ${ }^{4}$ Department of Human Genetics, University of Heidelberg, Heidelberg, Germany. ${ }^{5}$ Department of Rheumatology, University Hospital Heidelberg, Heidelberg, Germany. ${ }^{6}$ Division of Cardiology, U.O.C. Rehabilitazione Cardiovascolare, A Cardarelli, Naples, Italy. ${ }^{7}$ Department of Translational Medical Sciences, University Federico II of Naples, Naples, Italy. ${ }^{8}$ Cardiology Department, Royal Free Hospital, London, UK. ${ }^{9}$ Centre of Rheumatology, Royal Free Hospital, London, UK. ${ }^{10}$ Department of Rheumatology, University Hospital Zurich, Zurich, Switzerland.

\section{Received: 12 June 2019 Accepted: 16 August 2019} Published online: 26 October 2019

\section{References}

1. Condliffe R, Kiely DG, Peacock AJ, Corris PA, Gibbs JS, Vrapi F, et al. Connective tissue disease-associated pulmonary arterial hypertension in the modern treatment era. Am J Respir Crit Care Med. 2009;179(2):151-7.

2. Hao Y, Hudson M, Baron M, Carreira P, Stevens W, Rabusa C, et al. Early mortality in a multinational systemic sclerosis inception cohort. Arthritis Rheumatol. 2017:69(5):1067-77.

3. Condliffe R, Kiely DG, Gibbs JS, Corris PA, Peacock AJ, Jenkins DP, et al. Prognostic and aetiological factors in chronic thromboembolic pulmonary hypertension. Eur Respir J. 2009;33(2):332-8.

4. Kawut SM, Taichman DB, Archer-Chicko CL, Palevsky HI, Kimmel SE. Hemodynamics and survival in patients with pulmonary arterial hypertension related to systemic sclerosis. Chest. 2003;123(2):344-50.

5. Kovacs G, Avian A, Tscherner M, Foris V, Bachmaier G, Olschewski A, et al. Characterization of patients with borderline pulmonary arterial pressure. Chest. 2014;146(6):1486-93.

6. Visovatti SH, Distler O, Coghlan J, Denton CP, Grunig E, Bonderman D, et al. Borderline pulmonary arterial pressure in systemic sclerosis patients: a posthoc analysis of the DETECT study. Arthritis Res Ther. 2014;16(6):493.

7. Douschan P, Kovacs G, Avian A, Foris V, Gruber F, Olschewski A, et al. Mild elevation of pulmonary arterial pressure as a predictor of mortality. Am J Respir Crit Care Med. 2018;197(4):509-16.

8. Coghlan JG, Wolf M, Distler O, Denton CP, Doelberg M, Harutyunova S, et al. Incidence of pulmonary hypertension and determining factors in patients with systemic sclerosis. Eur Respir J. 2018;51(4).

9. Nagel C, Marra AM, Benjamin N, Blank N, Cittadini A, Coghlan G, et al. Reduced right ventricular output reserve in patients with systemic sclerosis and mildly elevated pulmonary arterial pressures. Arthritis Rheumatol. 2019; 71:805-16.

10. Simonneau G, Montani D, Celermajer DS, Denton CP, Gatzoulis MA, Krowka $M$, et al. Haemodynamic definitions and updated clinical classification of pulmonary hypertension. Eur Respir J. 2019;53(1).

11. Galie N, Olschewski H, Oudiz RJ, Torres F, Frost A, Ghofrani HA, et al. Ambrisentan for the treatment of pulmonary arterial hypertension: results of the ambrisentan in pulmonary arterial hypertension, randomized, doubleblind, placebo-controlled, multicenter, efficacy (ARIES) study 1 and 2. Circulation. 2008;117(23):3010-9.

12. Klinger JR, Oudiz RJ, Spence R, Despain D, Dufton C. Long-term pulmonary hemodynamic effects of ambrisentan in pulmonary arterial hypertension. Am J Cardiol. 2011;108(2):302-7.

13. Korn JH, Mayes M, Matucci Cerinic M, Rainisio M, Pope J, Hachulla E, et al. Digital ulcers in systemic sclerosis: prevention by treatment with bosentan, an oral endothelin receptor antagonist. Arthritis Rheum. 2004; 50(12):3985-93.

14. Matucci-Cerinic M, Denton CP, Furst DE, Mayes MD, Hsu VM, Carpentier P, et al. Bosentan treatment of digital ulcers related to systemic sclerosis: results from the RAPIDS-2 randomised, double-blind, placebo-controlled trial. Ann Rheum Dis. 2011;70(1):32-8.

15. Saggar R, Khanna D, Shapiro S, Furst DE, Maranian P, Clements $P$, et al. Brief report: effect of ambrisentan treatment on exercise-induced pulmonary hypertension in systemic sclerosis: a prospective single-center, open-label pilot study. Arthritis Rheum. 2012;64(12):4072-7.

16. Kovacs G, Maier R, Aberer E, Brodmann M, Graninger W, Kqiku X, et al. Pulmonary arterial hypertension therapy may be safe and effective in patients with systemic sclerosis and borderline pulmonary artery pressure. Arthritis Rheum. 2012;64(4):1257-62.

17. Humbert M, Yaici A, de Groote P, Montani D, Sitbon O, Launay D, et al. Screening for pulmonary arterial hypertension in patients with systemic sclerosis: clinical characteristics at diagnosis and long-term survival. Arthritis Rheum. 2011;63(11):3522-30.

18. Vizza CD, Fedele F, Pezzuto B, Rubin LJ. Safety and efficacy evaluation of ambrisentan in pulmonary hypertension. Expert Opin Drug Saf. 2012;11(6): 1003-11.

19. LeRoy EC, Medsger TA Jr. Criteria for the classification of early systemic sclerosis. J Rheumatol. 2001;28(7):1573-6.

20. Coghlan JG, Denton CP, Grunig E, Bonderman D, Distler O, Khanna D, et al. Evidence-based detection of pulmonary arterial hypertension in systemic sclerosis: the DETECT study. Ann Rheum Dis. 2014;73(7): 1340-9.

21. Kovacs G, Herve P, Barbera JA, Chaouat A, Chemla D, Condliffe R, et al. An official European Respiratory Society statement: pulmonary haemodynamics during exercise. Eur Respir J. 2017;50(5).

22. van den Hoogen F, Khanna D, Fransen J, Johnson SR, Baron M, Tyndall A, et al. 2013 classification criteria for systemic sclerosis: an American College of Rheumatology/European League against Rheumatism collaborative initiative. Arthritis Rheum. 2013;65(11):2737-47.

23. Galiè N, Humbert M, Vachiery JL, Gibbs S, Lang I, Torbicki A, et al. 2015 ESC/ ERS guidelines for the diagnosis and treatment of pulmonary hypertension: The Joint Task Force for the Diagnosis and Treatment of Pulmonary Hypertension of the European Society of Cardiology (ESC) and the European Respiratory Society (ERS): endorsed by: Association for European Paediatric and Congenital Cardiology (AEPC), International Society for Heart and Lung Transplantation (ISHLT). Eur Heart J. 2016;37(1):67-119.

24. Grünig E, Weissmann S, Ehlken N, Fijalkowska A, Fischer C, Fourme T, et al. Stress Doppler echocardiography in relatives of patients with idiopathic and familial pulmonary arterial hypertension: results of a multicenter European analysis of pulmonary artery pressure response to exercise and hypoxia. Circulation. 2009;119(13):1747-57.

25. Benza RL, Miller DP, Gomberg-Maitland M, Frantz RP, Foreman AJ, Coffey CS, et al. Predicting survival in pulmonary arterial hypertension: insights from the Registry to Evaluate Early and Long-Term Pulmonary Arterial Hypertension Disease Management (REVEAL). Circulation. 2010; 122(2):164-72.

26. Humbert M, Sitbon O, Chaouat A, Bertocchi M, Habib G, Gressin V, et al. Survival in patients with idiopathic, familial, and anorexigen-associated pulmonary arterial hypertension in the modern management era. Circulation. 2010;122(2):156-63.

27. Hsu S, Kokkonen-Simon KM, Kirk JA, Kolb TM, Damico RL, Mathai SC, et al. Right ventricular myofilament functional differences in humans with systemic sclerosis-associated versus idiopathic pulmonary arterial hypertension. Circulation. 2018;137(22):2360-70.

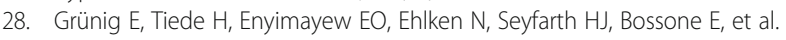
Assessment and prognostic relevance of right ventricular contractile reserve in patients with severe pulmonary hypertension. Circulation. 2013;128(18): 2005-15.

29. Chaouat A, Sitbon O, Mercy M, Poncot-Mongars R, Provencher S, Guillaumot $A$, et al. Prognostic value of exercise pulmonary haemodynamics in pulmonary arterial hypertension. Eur Respir J. 2014;44(3):704-13.

30. Shirai $Y$, Kuwana M. Complex pathophysiology of pulmonary hypertension associated with systemic sclerosis: potential unfavorable effects of vasodilators. J Scleroderm Rel Dis. 2017;2(2):92-9.

31. Chin KM, Bartolome S, Miller K, Blair C, Gillies H, Torres F. Does treatment response to ambrisentan vary by pulmonary arterial hypertension severity? Implications for clinicians and for the design of future clinical trials. Int J Clin Pract. 2014;68(5):568-77.

32. Galie N, Rubin L, Hoeper M, Jansa P, Al-Hiti H, Meyer G, et al. Treatment of patients with mildly symptomatic pulmonary arterial hypertension with bosentan (EARLY study): a double-blind, randomised controlled trial. Lancet. 2008;371(9630):2093-100.

33. Hachulla E, Launay D, Yaici A, Berezne A, de Groote P, Sitbon O, et al. Pulmonary arterial hypertension associated with systemic sclerosis in patients with functional class II dyspnoea: mild symptoms but severe outcome. Rheumatology (Oxford). 2010;49(5):940-4.

34. Takahashi T, Hayata S, Kobayashi A, Onaka Y, Ebihara T, Hara T. Surveillance on the safety and efficacy of ambrisentan (Volibris tablet $2.5 \mathrm{mg}$ ) in patients 
with pulmonary arterial hypertension in real clinical practice: post-marketing surveillance (interim analysis report). Clin Drug Investig. 2018;38(3):219-29.

35. Vachiery JL, Hoeper MM, Peacock AJ, Sitbon O, Cheli M, Church C, et al. Ambrisentan use for pulmonary arterial hypertension in a post-authorization drug registry: the VOLibris tracking study. J Heart Lung Transplant. 2017; 36(4):399-406.

36. Aversa M, Porter S, Granton J. Comparative safety and tolerability of endothelin receptor antagonists in pulmonary arterial hypertension. Drug Saf. 2015;38(5):419-35.

37. Oudiz RJ, Galie N, Olschewski H, Torres F, Frost A, Ghofrani HA, et al. Longterm ambrisentan therapy for the treatment of pulmonary arterial hypertension. J Am Coll Cardiol. 2009;54(21):1971-81.

38. Heresi GA, Minai OA, Tonelli AR, Hammel JP, Farha S, Parambil JG, Dweik RA. Clinical Characterization and Survival of Patients with Borderline Elevation in Pulmonary Artery Pressure. Pulm Circ. 2013;3(4):916-25

\section{Publisher's Note}

Springer Nature remains neutral with regard to jurisdictional claims in published maps and institutional affiliations.

Ready to submit your research? Choose BMC and benefit from:

- fast, convenient online submission

- thorough peer review by experienced researchers in your field

- rapid publication on acceptance

- support for research data, including large and complex data types

- gold Open Access which fosters wider collaboration and increased citations

- maximum visibility for your research: over $100 \mathrm{M}$ website views per year

At BMC, research is always in progress.

Learn more biomedcentral.com/submissions 